ARTICLE

\title{
The transcription factor ZIP-1 promotes resistance to intracellular infection in Caenorhabditis elegans
}

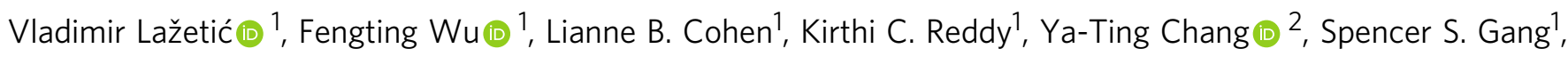
Gira Bhabha² \& Emily R. Troemel (10 ${ }^{1 凶}$

Defense against intracellular infection has been extensively studied in vertebrate hosts, but less is known about invertebrate hosts; specifically, the transcription factors that induce defense against intracellular intestinal infection in the model nematode Caenorhabditis elegans remain understudied. Two different types of intracellular pathogens that naturally infect the C. elegans intestine are the Orsay virus, which is an RNA virus, and microsporidia, which comprise a phylum of fungal pathogens. Despite their molecular differences, these pathogens induce a common host transcriptional response called the intracellular pathogen response (IPR). Here we show that zip-1 is an IPR regulator that functions downstream of all known IPR-activating and regulatory pathways. zip-1 encodes a putative bZIP transcription factor, and we show that zip-1 controls induction of a subset of genes upon IPR activation. ZIP-1 protein is expressed in the nuclei of intestinal cells, and is at least partially required in the intestine to upregulate IPR gene expression. Importantly, zip-1 promotes resistance to infection by the Orsay virus and by microsporidia in intestinal cells. Altogether, our results indicate that zip-1 represents a central hub for triggers of the IPR, and that this transcription factor has a protective function against intracellular pathogen infection in C. elegans.

\footnotetext{
${ }^{1}$ Division of Biological Sciences, University of California, San Diego, La Jolla, California, CA, USA. ${ }^{2}$ Department of Cell Biology, New York University School of

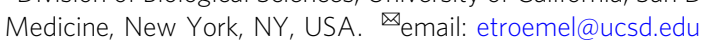


$\mathrm{V}$ iruses and other obligate intracellular pathogens are responsible for a myriad of serious illnesses ${ }^{1}$. RNA viruses, like the single-stranded, positive-sense RNA virus SARSCoV-2 that causes COVID-19, are detected by RIG-I-like receptors $^{2-4}$. These receptors detect viral RNA replication products and trigger transcriptional upregulation of interferon genes to induce antiviral defense ${ }^{5}$. The nematode Caenorhabditis elegans provides a simple model host to understanding responses to RNA viruses, as a single-stranded, positive-sense RNA virus from Orsay, France infects C. elegans in the wild ${ }^{6}$. Interestingly, natural variation in $d r h-1$, a $C$. elegans gene encoding a RIG-I-like receptor, was found to underlie natural variation in resistance to the Orsay virus ${ }^{7}$. Several studies indicate that detection of viral RNA by the $d r h-1$ receptor induces an antiviral response through regulating RNA interference $(\mathrm{RNAi})^{7-9}$.

In addition to regulating RNAi, $d r h-1$ detection of viral replication products was recently shown to activate a transcriptional immune/stress response in C. elegans called the intracellular pathogen response (IPR) ${ }^{10}$. The IPR was defined as a common transcriptional response to the Orsay virus and a molecularly distinct natural intracellular pathogen of $C$. elegans called Nematocida parisii ${ }^{11-13}$. N. parisii is a species of Microsporidia, which comprise a phylum of obligate intracellular fungal pathogens that infect a large range of animal hosts including humans. It is not known which host receptors detects $N$. parisii infection, but the DRH-1 RIG-I-like receptor appears to detect viral RNA replication products, and to be critical for viral induction of the IPR $^{10}$. Notably, C. elegans does not have clear orthologs of interferon, or the signaling factors that act downstream of RIG-Ilike receptors in mammals, such as the transcription factors NF$\mathrm{kB}$ and IRF3/714. It is unknown how $d r h-1$ activates the IPR transcriptional program in C. elegans.

Several noninfection inputs can also trigger IPR gene expression. For example, proteotoxic stress, such as that caused by blockade of the proteasome, or by prolonged heat stress, will induce IPR genes. While intracellular infection by the Orsay virus or by N. parisii cause hallmarks of proteotoxic stress in C. elegans intestinal cells ${ }^{11}$, genetic and kinetic analyses indicate that proteotoxic stress is activating IPR gene expression in parallel to viral infection, and that there are several independent triggers of the $I_{P R}{ }^{10}$. Another trigger of the IPR is mutation in the enzyme purine nucleoside phosphorylase-1, PNP-1, which acts in C. elegans intestinal epithelial cells to regulate pathogen resistance and the majority of IPR genes ${ }^{12,13,15}$. Of note, mutations in human PNP cause T-cell dysfunction, but its role in epithelial cells is less well-described $^{15}$. In addition to $p n p-1$, analysis of another IPR repressor called pals-22, has provided insight into the regulation and function of IPR genes ${ }^{12,13}$. pals-22 belongs to the pals (protein containing ALS2cr12 signature) gene family, which has one ortholog each in mouse and human of unknown function, while this family has expanded to 39 members in C. elegans ${ }^{12,16,17}$. The biochemical functions of pals genes are unknown, but they play important roles in intracellular infection in C. elegans ${ }^{13}$. Several pals genes (e.g., pals-5) are upregulated by virus infection and the other IPR triggers mentioned above. Furthermore, two pals genes, pals-22 and pals-25, are opposing regulators of the IPR, acting as an ON/OFF switch for IPR gene expression as well as resistance to infection ${ }^{13}$. Not only do pals-22 and pals- 25 control immunity, but they also control thermotolerance, a phenotype that is dependent on a subset of IPR genes that encode a newly described, multisubunit, E3 ubiquitin ligase that promotes proteostasis ${ }^{13,18}$.

While Orsay virus infection, N. parisii infection, proteotoxic stress, $p n p-1$ and pals-22 mutations all appear to act independently of each other to trigger IPR gene expression, here we show that they converge on a common downstream transcription factor. Using two RNAi screens, we find that the gene encoding a putative basic region-leucine zipper (bZIP) transcription factor called zip-1 plays a role in activating expression of the IPR gene pals -5 by all known IPR triggers. Furthermore, we use proteasome inhibition as a trigger to show that zip-1 controls induction of only a subset of IPR genes. These results demonstrate that there are at least three classes of IPR genes as defined by whether their induction is dependent on zip-1 early after proteasome inhibition, late after proteasome inhibition, or their induction after proteasome inhibition is independent of zip-1. We show that the ZIP1::GFP protein expression is induced in intestinal and epidermal nuclei upon IPR activation, and that ZIP-1 likely functions in the intestine to activate pals-5 expression. We find that induction of ZIP-1::GFP expression in intestinal nuclei by viral infection depends on DRH-1, suggesting that the DRH-1 receptor controls activation of the ZIP-1 transcription factor. Importantly, we show that $z i p-1$ promotes defense against viral as well as against microsporidia infection in the intestine. Altogether, our results define zip-1 as a central signaling hub, controlling induction of IPR gene expression in response to a wide range of triggers, including diverse intracellular pathogens, other stressors, and genetic regulators. Furthermore, this study describes ZIP-1 as the first transcription factor shown to promote an inducible defense response against intracellular intestinal infection in C. elegans.

\section{Results}

Two independent screens for regulators of the IPR identify the predicted transcription factor ZIP-1. To determine which transcription factor(s) activates IPR gene expression, we screened an RNAi library composed of 363 RNAi clones targeting 357 predicted transcription factors (less than half the predicted transcription factor repertoire in C. elegans) to identify RNAi clones that repress constitutive expression of the PALS-5::GFP translational reporter (jyEx191) in a pals-22(jy3) background. In parallel, we also screened this library for RNAi clones that prevent induction of the pals-5p::GFP transcriptional reporter (jyIs8) upon prolonged heat stress. In both screens, we found that zip1(RNAi) led to a substantial decrease in GFP signal (Fig. 1a, b, Supplementary Data 1), suggesting that this putative bZIPcontaining transcription factor plays a role in IPR regulation. We confirmed this zip-1(RNAi) phenotype in another pals-22 loss-offunction allele, $j y 1$, showing that here too, RNAi against zip-1 repressed the constitutive expression of PALS-5::GFP in pals-22 mutants (Fig. 1c). To demonstrate that this phenotype is not just restricted to $z i p-1(R N A i)$, we created a full deletion allele of $z i p-1$ called jy13 (Supplementary Fig. 1), and observed decreased expression of the pals-5p::GFP reporter following prolonged heat stress in this putative zip-1 null mutant (Fig. 1d). These results indicate that $z i p-1$ is important for regulating expression of two different pals-5 GFP reporters by two different IPR triggers.

We also specifically examined whether RNAi against two other transcription factors implicated in intracellular infection were required to induce pals-5p::GFP expression. First, we analyzed the role of bZIP transcription factor zip-10, which is induced as part of the IPR and promotes N. parisii sporulation ${ }^{13,19}$. Here we found no effect of zip-10(RNAi) on pals-5p::GFP induction after chronic heat stress. We also analyzed the role of STAT-like transcription factor sta-2, which is important for response to Drechmeria coniospora, a fungal pathogen that penetrates and grows inside epidermal cells ${ }^{20,21}$. Here as well, we did not find an effect of sta-2(RNAi) on induction of pals-5p::GFP expression (Supplementary Fig. 2).

zip-1 is required for induction of pals-5p::GFP expression by all known IPR triggers. We also investigated whether $z i p-1$ was required for inducing pals-5p::GFP expression upon other IPR 

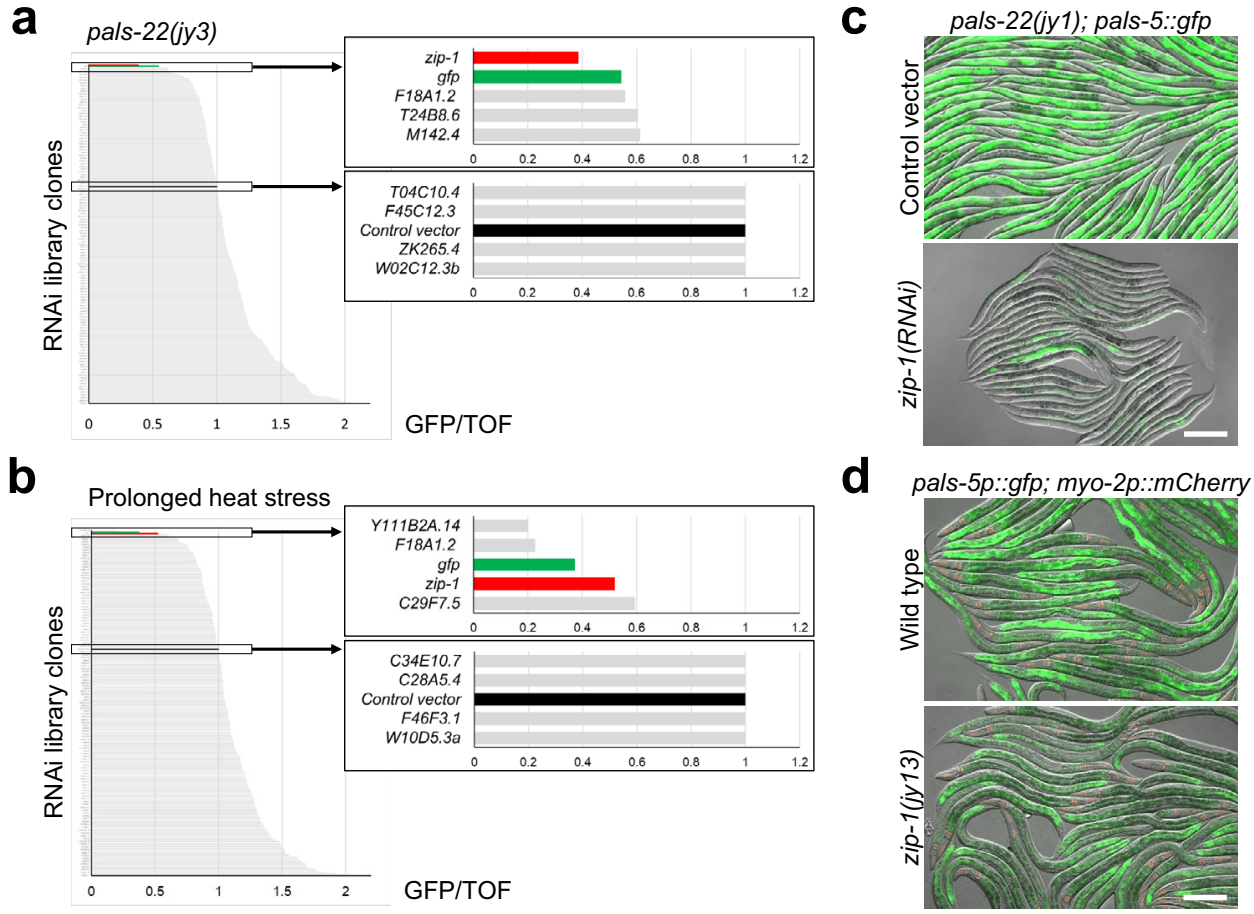

Fig. 1 zip-1 is required for induction of pals-5 GFP reporters by pals-22(RNAi) and by prolonged heat stress. a, b Graphical overview of RNAi screen results in the pals-22(jy3); jyEx191[pals-5::gfp] background (a) and following chronic heat stress (b). GFP intensity was normalized to the length of worms (TOF) and it is indicated on the $x$-axis; different RNAi clones are listed on the $y$-axis. Boxes on the right represent enlarged sections of the graph containing zip-1(RNAi) results and relevant controls. Source data are provided as a Supplementary Data 1 file. c pals-22(jy1); jyEx191[pals-5::gfp] animals show constitutive expression of the PALS-5::GFP reporter when grown on control vector RNAi plates (upper image) but not on zip-1 RNAi plates (lower image). Two independent experimental replicates were performed with similar results. $\mathbf{d}$ Expression of GFP from the jyls8[pals-5p::gfp, myo-2p::mCherry] reporter is decreased in zip-1(jy13) animals following prolonged heat stress (lower image), in comparison to wild-type animals (upper image). Three independent experimental replicates were performed with similar results. c, d Fluorescent and DIC images were merged. Scale bars $=200 \mu \mathrm{m}$. myo-2p::mCherry is expressed in the pharynx and is a marker for the presence of the jyls 8 transgene.

triggers. First, we tested whether zip-1 was required for response to infection with the Orsay virus. Here we found that while infection of wild-type animals with the Orsay virus induced expression of the pals-5p::GFP reporter, infection of zip-1(jy13) mutants caused no GFP induction (Fig. 2a). Similarly, infection with the microsporidian species $N$. parisii caused GFP expression throughout intestine in wild-type animals, but little to no GFP expression in $z i p-1(j y 13$ ) mutants (Fig. 2b). Therefore, $z i p-1$ is required for induction of pals-5p::GFP expression after infection by these two natural intracellular pathogens of the C. elegans intestine. We next tested if $z i p-1$ was required for constitutive expression of pals-5p::GFP in pnp-1 mutants ${ }^{15}$. Here, we also saw a requirement for $z i p-1$, as zip-1(jy13); pnp-1(jy90) double mutants had much less pals-5p::GFP signal compared to $p n p-1$ single mutants (Fig. 2c, d). Finally, we investigated whether zip-1 is required for induction of pals-5p::GFP using the drug bortezomib $^{10,11,18,22}$. Here we also saw that $z i p-1$ was required for induction of pals-5p::GFP across a timecourse of bortezomib treatment (Fig. 2e, f). Therefore, of the six IPR triggers we tested, $z i p-1$ was required for induction of pals-5p::GFP by all of them.

Because the jyIs8[pals-5p:::fp] and jyEx191[pals-5::gfp] reporters described above are multi-copy transgene arrays that could be prone to silencing, we considered the possibility that zip-1 repressed GFP expression in the previous experiments through its effects on transgene silencing. Therefore, we next investigated if zip-1 inhibited expression from a single-copy transcriptional reporter, as single-copy transgenes are much less prone to silencing than multi-copy arrays. Here we used the strain with a single-copy jySi44[pals-5p::NanoLuc] transgene insertion, where the pals-5 promoter drives expression of the bioluminescent protein nanoluciferase 22 . Here we also found that $z i p-1$ was required for induction of pals-5p::NanoLuc bioluminescence by proteasome blockade (Fig. 2g), further indicating that zip-1 regulates gene expression driven by the pals-5 promoter.

Because the zip-1 genomic locus contains a noncoding RNA $y 75 b 8 a .55$ in one of its introns, and this noncoding RNA is also deleted in the $z i p-1(j y 13)$ deletion strain, we also created a partial deletion allele of zip-1 called jy14 (Supplementary Fig. 1). This $y 75 b 8 a .55$ noncoding RNA locus is preserved in the jy14 allele, while the region encoding the predicted bZIP domain of zip- 1 is deleted. Here, with intracellular infection and with proteasome blockade treatment, we also found that pals-5p::GFP reporter expression was much lower or absent in zip-1(jy14) mutants than in wild-type animals, and indistinguishable from the phenotype of $z i p-1(j y 13)$ mutants (Supplementary Fig. 3). In summary, we found that phenotypes observed after loss of zip-1 are not allelespecific and that they likely cannot be attributed to inactivation of the $y 75 b 8 a .55$ gene. Altogether, these results indicate that zip-1 controls expression of pals- 5 reporters induced by all wellcharacterized triggers of IPR gene expression.

$z i p-1$ is required for early induction of pals-5 mRNA as well as induction of a subset of other IPR genes. We next used qRTPCR to assess the role of zip-1 in controlling levels of endogenous pals-5 mRNA, as well as mRNA of other IPR genes. Because bortezomib treatment induced the strongest and most consistent IPR gene expression, we used this trigger to assess the role of zip1 in mediating IPR gene induction in subsequent experiments. Here we were surprised that $z i p-1(j y 13)$ mutants had only about a 
a

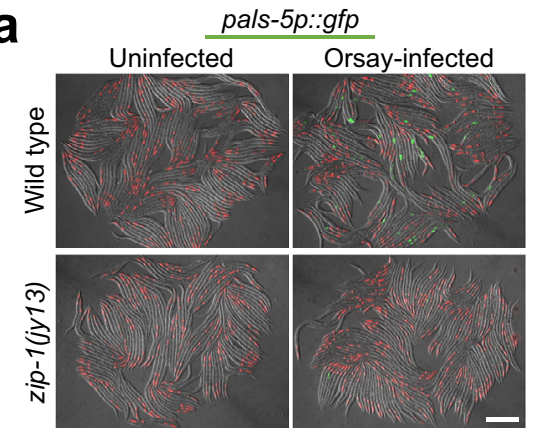

C

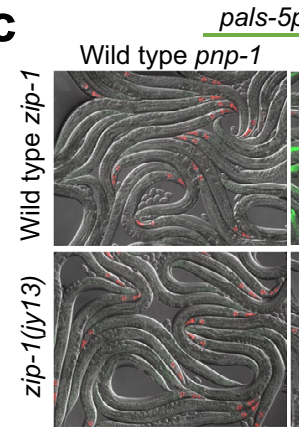
pnp-1(-)

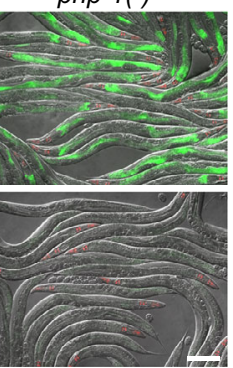

b

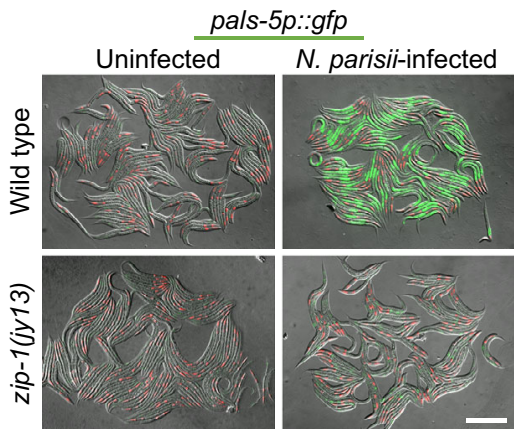

e

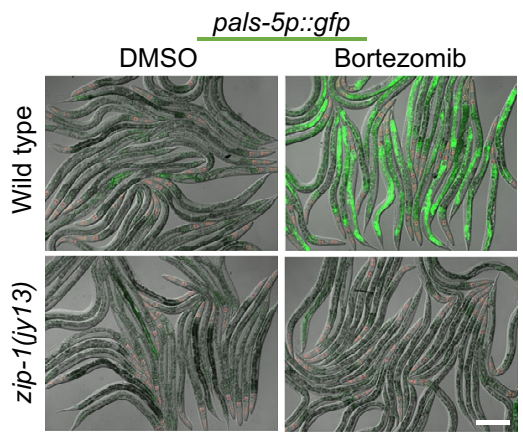

d

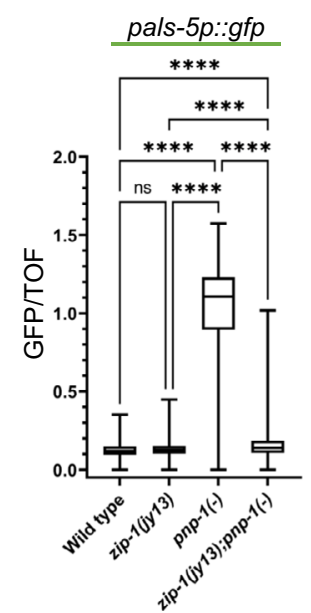

f

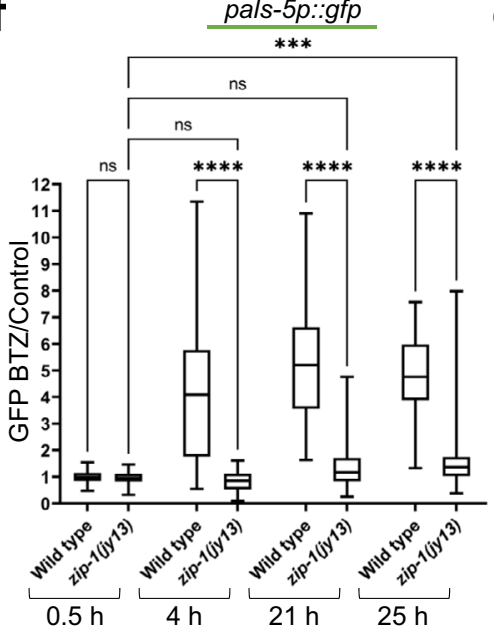

$9 \quad$ pals-5p::NanoLuc

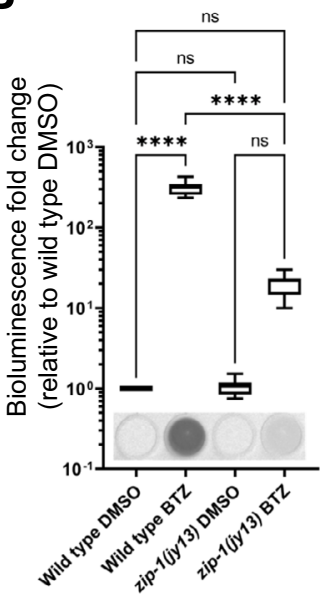

Fig. 2 zip-1 is required for induction of pals-5p::GFP expression by intracellular infections, pnp-1 downregulation, and proteasome blockade.

a, b Intracellular infection by Orsay virus (a) and by N. parisii (b) leads to pals-5p::GFP expression in wild-type animals, but not in zip-1(jy13) mutants. c pnp1(jy90) mutants show constitutive expression of the pals-5p::GFP reporter, which is suppressed in zip-1(jy13); pnp-1(jy90) double mutants. d Box-andwhisker plot of pals-5p::GFP expression normalized to length of animals (TOF). Increased GFP signal in pnp-1(jy90) mutants is significantly reduced in zip1(jy13); pnp-1(jy90) double mutants. Three experimental replicates with 400 animals per replicate were analyzed for each strain. e Bortezomib treatment induces expression of pals-5p::GFP in a wild-type background, but not in zip-1(jy13) mutants. a-c, e Three independent experimental replicates were performed with similar results. Fluorescent and DIC images were merged. Scale bars $=200 \mu \mathrm{m}$. myo-2p::mCherry is expressed in the pharynx and is a marker for the presence of the jyls8 transgene. $\mathbf{f}$ Timecourse analysis of pals-5p::GFP expression in control and zip-1(jy13) strains following bortezomib treatment. GFP signal normalized to worm area is shown as a fluorescence intensity ratio between bortezomib- and DMSO-treated samples ( $y$-axis). Three experimental replicates with 30 animals per replicate were analyzed; average value was used for DMSO controls. Allele names and timepoints of analysis are indicated on the $x$-axis. $\mathbf{g}$ Expression of the pals-5p::NanoLuc reporter is significantly lower in zip-1(jy13) animals than in the wild-type control strain, following bortezomib treatment. Three experimental replicates consisting of three biological replicates were analyzed for each strain and treatment. Results were normalized to background luminescence and to average value of three biological replicates for wild-type treated with DMSO. Normalized relative luminescent units (RLU) are shown on the $y$-axis. Images of bioluminescent signal in representative analyzed wells are shown on the bottom of the graph. $\mathbf{d}, \mathbf{f}, \mathbf{g}$ In box-and-whisker plots, the line in the box represents the median value, box bounds indicate the 25th and 75th percentiles, and whiskers extend from the box bounds to the minimum and maximum values. A Kruskal-Wallis test (d, $\mathbf{f}$ ) or ordinary one-way ANOVA test ( $\mathbf{g}$ ) were used to calculate $p$ values; ${ }^{\star \star \star \star} p<0.0001 ;{ }^{\star \star \star} p<0.001$; ns indicates nonsignificant difference $(p>0.05)$. $p$-values in d for WT vs zip-1(jy13) $p=0.3552$, other comparisons $p<0.0001$. $p$-values in f for WT vs zip-1(jy13) at $0.5 \mathrm{~h} \mathrm{p}>0.9999$; WT vs zip-1(jy13) at 4,21 and $25 \mathrm{~h} \mathrm{p}<0.0001 ;$ zip-1(jy13) $0.5 \mathrm{~h}$ vs zip-1(jy13) $4 \mathrm{~h}$ $p>0.9999 ;$ zip-1(jy13) 0.5 h vs zip-1(jy13) $21 \mathrm{~h} p=0.1093$; zip-1(jy13) $0.5 \mathrm{~h}$ vs zip-1(jy13) $25 \mathrm{~h} p=0.0004$. $p$-values in g for WT DMSO vs WT BTZ $p<0.0001$; WT DMSO vs zip-1(jy13) DMSO $p>0.9999 ;$ WT DMSO vs zip-1(jy13) BTZ $p=0.5576$; WT BTZ vs zip-1(jy13) BTZ $p<0.0001 ;$ zip-1(jy13) DMSO vs zip-1(jy13) BTZ $p=0.5601$. Source data are provided as a Source Data file. 

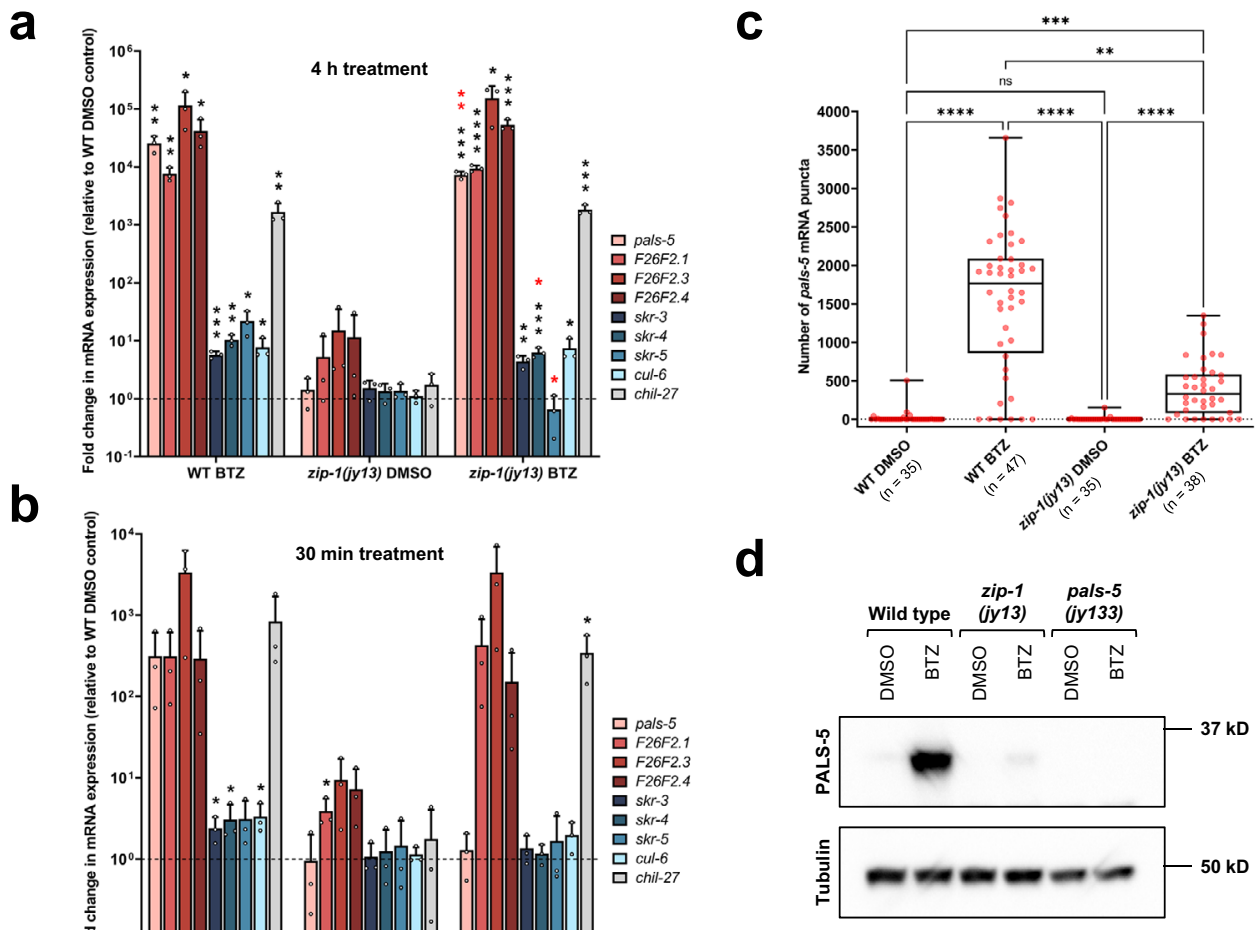

Fig. 3 zip-1 regulates the early phase of pals- 5 transcription following bortezomib treatment, and controls some IPR gene expression. a, b $q R T-P C R$ measurements of selected IPR genes and chil-27 at $4 \mathrm{~h}$ timepoint (a) and 30 min timepoint (b) of bortezomib (BTZ) or DMSO treatments. The results are shown as the fold change in gene expression relative to wild-type DMSO diluent control. Three independent experimental replicates were analyzed; the values for each replicate are indicated with circles. Bar heights indicate mean values and error bars extend above. Error bars represent standard deviations. A one-tailed $t$-test was used to calculate $p$-values; black asterisks represent significant difference between the labeled sample and the wild-type DMSO control; red asterisks represent significant difference between zip-1(jy13) and wild-type (WT) N2 bortezomib-treated samples; ${ }^{\star \star \star \star} p<0.0001 ;{ }^{* \star \star} p<0.001$; ${ }^{*} p<0.01 ;{ }^{*} 0.01<p<0.05 ; p$-values higher than 0.05 are not labeled. $p$-values in a for WT BTZ vs WT DMSO: pals-5 $p=0.0026, F 26 F 2.1 p=0.0017$, F26F2.3 $p=0.0343$, F26F2.4 $p=0.0211$, skr-3 $p=0.0003$, skr-4 $p=0.0012$, skr-5 $p=0.0132$, cul-6 $p=0.0117$, chil-27 $p=0.0070 ;$ zip-1(jy13) BTZ vs WT DMSO: pals-5 $p=0.0002$, F26F2.1 $p<0.0001$, F26F2.3 $p=0.0224$, F26F2.4 $p=0.0009$, skr-3 $p=0.0030$, skr-4 p=0.0009, cul-6 $p=0.0159$, chil-27 $p=0.0005$; zip-1(jy13) BTZ vs WT BTZ: pals-5 $p=0.0086$, skr-4 $p=0.0287$, skr-5 $p=0.0126$. $p$-values in b for WT BTZ vs WT DMSO: skr-3 $p=0.0258$, skr-4 $p=0.0477$, cul-6 $p=0.0248$; zip-1(jy13) DMSO vs WT DMSO: F26F2.1 $p=0.0192 ;$ zip-1(jy13) BTZ vs WT DMSO: chil-27 $p=0.0262$. All significant and nonsignificant $p$-values are provided as a Source Data file. c smFISH quantification of number of pals-5 mRNA transcripts in the first four intestinal cells. Three experimental replicates were performed. At least 33 animals were analyzed for each sample (WT DMSO $n=35$, WT BTZ $n=47$, zip-1(jy13) DMSO $n=35$, zip-1(jy13) BTZ $n=38$; at least five animals were analyzed per sample per replicate) $4 \mathrm{~h}$ after bortezomib or DMSO control treatment. In box-andwhisker plots, the line in the box represents the median value, box bounds indicate the 25th and 75th percentiles, and whiskers extend from the box bounds to the minimum and maximum values. A Kruskal-Wallis test was used to calculate $p$-values; ${ }^{* \star \star \star} p<0.0001 ;{ }^{\star \star \star} p<0.001 ;{ }^{\star \star} p<0.01$; ns indicates nonsignificant difference ( $p>0.05)$. $p$-values for WT DMSO vs WT BTZ, WT BTZ vs zip-1(jy13) DMSO and zip-1(jy13) DMSO vs zip-1(jy13) BTZ p<0.0001; WT DMSO vs zip-1(jy13) DMSO $p>0.9999 ;$ WT DMSO vs zip-1(jy13) BTZ $p=0.0001$; WT BTZ vs zip-1(jy13) BTZ $p=0.0037$. d Western blot analysis of PALS-5 expression in wild-type, zip-1(jy13) and pals-5(jy133) animals. pals-5(jy133) is a complete deletion of the pals-5 gene and was used as a negative control. Animals were treated with bortezomib or DMSO control for $4 \mathrm{~h}$. PALS-5 was detected using anti-PALS-5 antibody, whereas anti-tubulin antibody was used as a loading control. Predicted sizes are 35.4 kD for PALS-5 and around $50 \mathrm{kD}$ for different members of the tubulin family. One out of three independent experimental replicates is shown; similar results were obtained from all three replicates (other two replicates are shown in the Supplementary Fig. 5a). a-d Source data are provided as a Source Data file.

3.5-fold reduction in pals-5 mRNA induction at $4 \mathrm{~h}$ after bortezomib treatment compared to induction in wild-type animals (Fig. 3a). Four hours is the timepoint at which zip-1 mutants were strongly defective for induction of pals-5p::GFP and pals5p::NanoLuc expression (Fig. 2e-g). Therefore, we considered the possibility that GFP and nanoluciferase expression observed at $4 \mathrm{~h}$ may reflect protein synthesized from mRNA made earlier. To investigate this possibility, we used qRT-PCR to measure pals5 mRNA at 30 minutes ( $\mathrm{min}$ ) after bortezomib treatment, and here we found that zip-1 was completely required for the $\sim 300$ fold induction of pals-5 mRNA at this early timepoint (Fig. 3b). Thus, zip-1 is completely required for pals-5 mRNA induction $30 \mathrm{~min}$ after bortezomib treatment, but only partially required for induction at $4 \mathrm{~h}$ after bortezomib treatment.
Because zip-1 appeared to be more important at $4 \mathrm{~h}$ for inducing GFP and nanoluciferase transcriptional reporters than for inducing pals-5 mRNA by qRT-PCR, we used smFISH as a separate measure for pals-5 mRNA levels at this timepoint. Here, as in the GFP reporter studies, pals-5 expression was seen in the intestine. Because it is an easily identified location, we quantified pals-5 RNA levels in the first intestinal ring, which is comprised of four epithelial cells. Here we found that pals-5 mRNA was induced to a lesser degree in zip-1 mutants treated with bortezomib compared to wild-type animals (Fig. 3c, Supplementary Fig. 4).

Next, to determine whether zip-1 mutants are defective in PALS-5 protein production, we raised polyclonal antibodies against the PALS-5 protein. Using these antibodies for western 
blots, we found that PALS-5 protein induction in zip-1(jy13) animals at $4 \mathrm{~h}$ after bortezomib treatment was almost undetectable in comparison to the induction in bortezomib-treated wildtype animals (Fig. 3d, Supplementary Fig. 5). Therefore, zip-1 is required for high levels of PALS-5 protein production after bortezomib treatment, very likely through its role in regulating induction of pals-5 mRNA.

Having confirmed that zip-1 is completely required for induction of pals-5 mRNA at $30 \mathrm{~min}$ and partially required at $4 \mathrm{~h}$, we examined the requirement for $z i p-1$ in induction of other IPR genes at these timepoints. We analyzed highly induced IPR genes of unknown function - F26F2.1, F26F2.3, and F26F2.4, as well as components of a cullin-RING ubiquitin ligase complex cul-6, skr-3, skr-4 and skr-5, which mediates thermotolerance as part of the IPR program ${ }^{18}$. Interestingly, zip-1 was not required at either timepoint ( $30 \mathrm{~min}$ or $4 \mathrm{~h}$ after bortezomib treatment) for mRNA induction of the majority of genes we analyzed, including F26F2.1 (Fig. 3a, b). In agreement with these results, zip-1 was not required for F26F2.1p::GFP expression after bortezomib treatment (Supplementary Fig. 6). Furthermore, zip-1 was not required for induction of the chitinase-like gene chil-27, which is induced by bortezomib, as well as by the natural oomycete pathogen Myzocytiopsis humicola ${ }^{13,23}$. In contrast, zip-1 was required at the $4 \mathrm{~h}$ timepoint for induction of $s k r-5 \mathrm{mRNA}$ levels (Fig. 3b). Because the induction of $s k r-5$ at $30 \mathrm{~min}$ was quite low, it was difficult to assess the role of $z i p-1$ in regulating this gene at this timepoint. Overall, these results suggest that there are at least three classes of IPR genes: (1) genes that require zip-1 for early but not later induction ("Early zip-1-dependent" genes like pals5), (2) genes that require $z i p-1$ at the later timepoint ("Late zip-1dependent" genes like $s k r-5$ ), and (3) genes that do not require $z i p-1$ at either timepoint for their induction ("zip-1-independent" genes like F26F2.1).

We also analyzed the role of $z i p-1$ for induction of IPR gene expression upon intracellular infection (Supplementary Fig. 7). Similar to the results using bortezomib as a trigger, we found that $z i p-1$ was required for induction of pals- 5 mRNA expression by $N$. parisii infection and by Orsay virus infection, but was not required for induction of F26F2.1 mRNA expression by infection with these pathogens. However, zip-1 was only partially required for $s k r-5$ mRNA expression induction following Orsay-virus infection, in contrast to its complete requirement for $s k r-5$ mRNA expression upon bortezomib treatment, as described above. Of note, $s k r-5$ mRNA expression was not highly induced upon $N$. parisii infection, in either wild-type animals or in zip-1 mutants (Supplementary Fig. 7).

RNA sequencing analysis reveals a genome-wide picture of $z i p$ 1-dependent genes. To obtain a genome-wide picture of the genes controlled by $z i p-1$, we next performed RNA sequencing (RNA-seq) analysis. Here we treated wild-type N2 or zip-1(jy13) mutant animals with either bortezomib or vehicle control for either $30 \mathrm{~min}$ or $4 \mathrm{~h}$, then collected RNA and performed RNAseq. Based on differential expression analyses, we created lists of genes upregulated in each genetic background after bortezomib treatment at both analyzed timepoints. At $30 \mathrm{~min}$, we found that 136 and 215 genes were upregulated in wild-type and zip-1(jy13) animals, respectively, with 72 genes being upregulated in both backgrounds (Fig. 4a, Supplementary Data 2). Therefore, 64 genes (i.e., 136 minus 72 genes) were induced only in wild-type animals, indicating that their induction is zip-1-dependent early upon proteasome blockade. Importantly, pals-5 was among these genes that were only upregulated in wild-type animals and not zip-1 mutants at this timepoint, consistent with our qRT-PCR analysis (Fig. 3b). At $4 \mathrm{~h}$, we identified many more genes that showed differential expression between bortezomib and control treatments in both genetic backgrounds, with 2923 and 2813 genes upregulated in wild-type and zip-1(jy13) mutants, respectively (Fig. 4b, Supplementary Data 2). 2035 genes were upregulated in both backgrounds, meaning that 888 genes (2923 minus 2035 genes) were specifically upregulated in wild-type animals. 883 out of 888 genes belong to the "Late zip-1-dependent" category, and include $s k r-5$, consistent with our qRT-PCR analysis (Fig. 3a). Notably, five genes (ZK355.8, K02E7.10, math-39, gst-33, and F55G1.7) were induced only in wild-type animals at both examined timepoints, and thus we classified these genes as "Completely zip-1-dependent". Therefore, 59 (64 minus 5) genes from the 30 min timepoint belong to the "Early zip-1-dependent" genes category. Of note, consistent with our qRT-PCR and GFP reporter analyses, the F26F2.1 gene was upregulated in both genetic backgrounds following bortezomib treatment, and thus belongs to the "zip-1-independent" category.

We next examined the correlation between zip-1-dependent genes (separately analyzing genes induced at each timepoint) and genesets that were previously associated with IPR activation. Here we found that there is a significant similarity between zip-1dependent genes induced after $30 \mathrm{~min}$ bortezomib treatment, and genes upregulated early after Orsay virus infection, $N$. parisii infection, ectopic expression of Orsay viral RNA1, and genes induced in pals-22 and pnp-1 mutants (Fig. 4c, Supplementary Data 3 ). In addition, there is a significant similarity between genes induced at $30 \mathrm{~min}$ timepoint and canonical IPR genes. Similarly, there is a significant overlap between zip-1-dependent genes induced after $4 \mathrm{~h}$ bortezomib treatment and the majority of these IPR-associated genesets. Of note, there was not a significant overlap between zip-1-dependent genes induced after $4 \mathrm{~h}$ bortezomib treatment, and genes that are upregulated at the late phases of viral (96 hpi) and microsporidia infections (40 hpi and $60 \mathrm{hpi}$ ). These results suggest that $z i p-1$ plays a more important role in the acute transcriptional response to intracellular infection, and perhaps a lesser role later in infection. Furthermore, our analysis revealed significant similarity between zip-1upregulated genes and genes that are downregulated by sta-1. STA-1 is a STAT-related transcription factor that acts as a negative regulator of IPR gene expression. (Supplementary Fig. 8a, Supplementary Data 3). We also found a significant overlap between zip-1-dependent genes and those induced by $M$. humicola, a natural oomycete pathogen that infects the epidermis, although zip-1 was not required for induction of the chitinase-like gene chil-27, which is a common marker for M. humicola response (Supplementary Fig. 8b, Supplementary Data 3). Previous studies have shown connections between the IPR and genes induced either by $M$. humicola infection, or by extract from $M$. humicola as part of the oomycete recognition response in the epidermis ${ }^{13,23,24}$

We identified zip-1-dependent genes in our analysis here using proteasome blockade by bortezomib, which has effects on transcription that are unrelated to the IPR. For example, bortezomib activates the bounceback response that induces expression of proteasome subunits, and it is controlled by the conserved transcription factor SKN-1/Nrf2 25 . Therefore, we compared if zip-1-dependent genes (from both analyzed timepoints) have significant overlap with $s k n$-1-dependent genes. Here we found no significant similarity between the majority of analyzed datasets (Supplementary Fig. 8c, Supplementary Data 3). These results are consistent with previous IPR RNA-seq studies showing a distinction between the IPR and the bounceback response, and suggest that zip-1 does not play a role in the bounceback response ${ }^{11,13}$.

In addition, we found that zip-1 mRNA itself was strongly upregulated in wild-type animals following bortezomib treatment, 
a

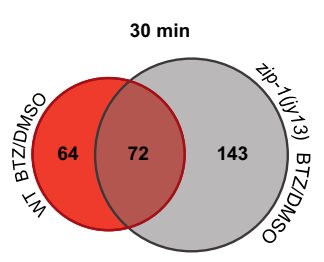

b

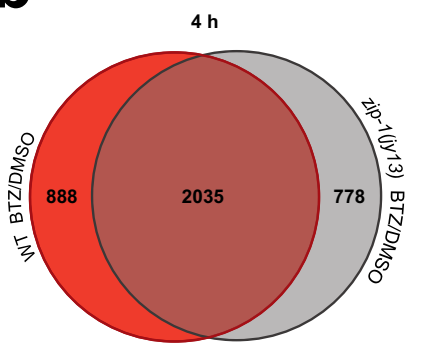

d

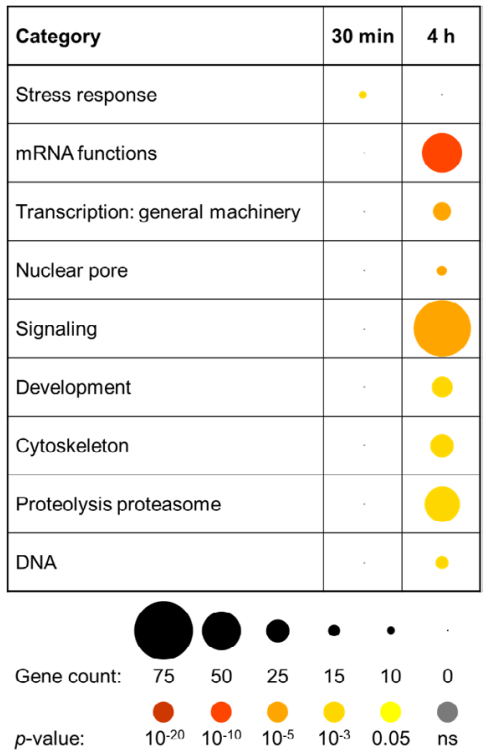

e

\begin{tabular}{|c|c|c|c|c|}
\hline \multirow{2}{*}{$\begin{array}{c}\text { Early } \\
\text { zip-1- } \\
\text { dependent }\end{array}$} & \multirow{2}{*}{$\begin{array}{c}\text { Late } \\
\text { zip-1- } \\
\text { dependent }\end{array}$} & \multicolumn{2}{|c|}{ zip-1-independent } & \multirow{2}{*}{ Not induced } \\
\hline & & Class A & Class B & \\
\hline$\overline{B 0507.6}$ & C08E3.13 & $B 0507.8$ & $\overline{B 0507.7}$ & clec-86 \\
\hline С53A5.11 & F49H6.5 & F15B9.6 & B0507.9 & F57B9.3 \\
\hline C53A5.9 & $f b x a-86$ & F26F2.1 & C43D7.4 & his-11 \\
\hline$d d n-1$ & math-38 & $h s p-17$ & clec- 60 & his-15 \\
\hline droe-8 & skr-5 & M01G12.9 & dod-23 & his-44 \\
\hline eol-1 & Y119D $\overline{3 B .21}$ & math-14 & F07E5.9 & pals-12 \\
\hline F42C5.3 & Y68A4A.13 & math-5 & $F 26 D 11.13$ & Y $41 C 4 A .11$ \\
\hline$f b x a-182$ & & pals-32 & F26F2.4 & Y69A2AR. 25 \\
\hline pals-14 & & pals-7 & F57G4.11 & \\
\hline pals-2 & & T08E11.1 & $f b x a-158$ & \\
\hline pals-26 & & T10C6.15 & $f b x a-165$ & \\
\hline pals-27 & & T24E12.5 & fbxa-75 & \\
\hline pals-28 & & Y75B8A.39 & math-10 & \\
\hline pals-30 & & Y94H6A.2 & pals-11 & \\
\hline pals-33 & & & pals-29 & \\
\hline pals-37 & & & pals-3 & \\
\hline pals-39 & & & pals-36 & \\
\hline pals-5 & & & pals-38 & \\
\hline pals-6 & & & pals-4 & \\
\hline Y2H9A. 6 & & & pals-8 & \\
\hline Y39G8B.5 & & & pals-9 & \\
\hline Y46G5A.20 & & & $p g p-8$ & \\
\hline Y $6 E 2 A .5$ & & & skr-4 & \\
\hline & & & T05A8.2 & \\
\hline & & & Y37H2A.14 & \\
\hline & & & Y47H10A.5 & \\
\hline & & & Y6E2A.7 & \\
\hline & & & ZC196.3 & \\
\hline
\end{tabular}

C
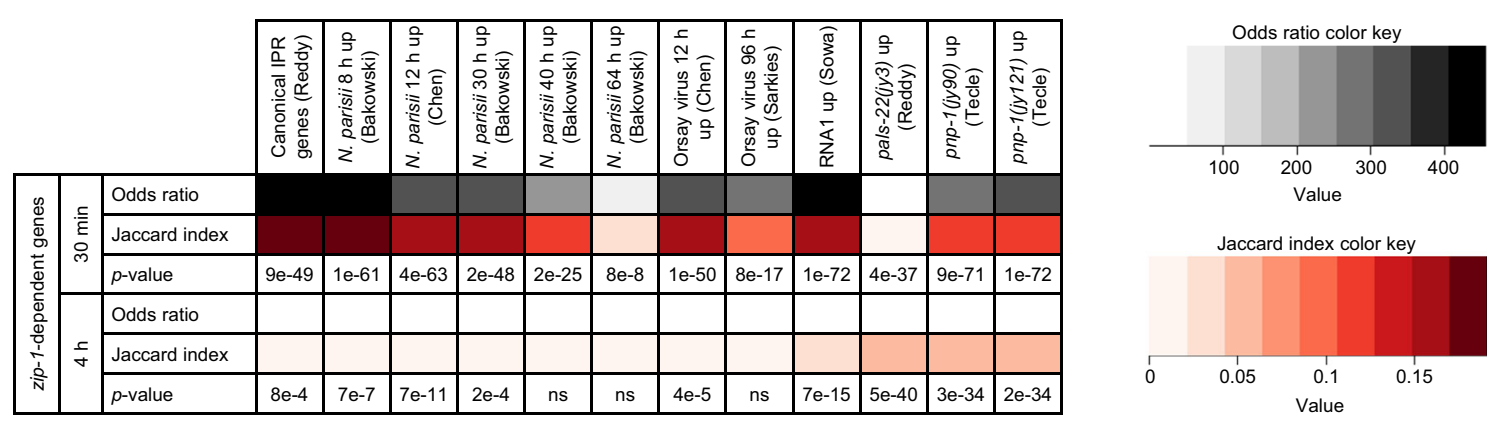

Fig. 4 Defining zip-1-dependent IPR genes. a, b Venn diagrams of differentially expressed genes following $30 \mathrm{~min}$ (a) and $4 \mathrm{~h}$ bortezomib treatments (b) in WT N2 and zip-1(jy13) mutant animals as compared to DMSO controls for each background. 64 and 888 genes were upregulated after 30 min and $4 \mathrm{~h}$ bortezomib treatment in N2 animals, respectively, but not in zip-1(jy13) mutants, suggesting that these genes are zip-1-dependent. Source data are provided as a Supplementary Data 2 file. c The zip-1-dependent gene set shows significant overlap with previously published list of genes that are upregulated by different IPR triggers. A Fisher's exact test was used to calculate odds ratios and $p$-values. These values were calculated taking in account all genes in $C$. elegans genome. If the odds ratio is greater than one, two datasets are positively correlated. Jaccard index measures similarity between two sets, with the range 0-1 (0 - no similarity, 1 - same datasets). For approximate quantification, the odds ratio and Jaccard index color keys are indicated on the right side of the table. Source data are provided as a Supplementary Data 3 file. $\mathbf{d}$ Bubble plot of enriched gene categories for all zip-1-dependent genes at 30 min and $4 \mathrm{~h}$ timepoints of bortezomib treatment. Each category represents a biological process or a structure associated with zip-1-dependent genes at either timepoint. Count of genes found in each category is indicated by the circle size, as illustrated under the table. Statistical significance for each category is indicated by the circle color; $p$-values are indicated under the table. $p$-values were determined using Bonferroni correction from the minimum hypergeometric scores calculated by the WormCat software. Source data (including p-values) are provided as a Supplementary Table 1. e Classification of 80 canonical IPR genes based on their zip-1 dependency. Representative canonical IPR genes from each class are shown in bold and underlined. Source data are provided as a Supplementary Data 2 file.

consistent with previous studies ${ }^{13}$. Surprisingly, however, we found that zip-1 mRNA was also upregulated in zip-1 mutants. This result that was initially confusing, because the $z i p-1$ coding sequence is completely deleted in the zip-1(jy13) allele that we used in RNA-seq analysis. Upon closer examination, however, we found that $z i p-1$ sequencing reads in $z i p-1(j y 13)$ mutant samples aligned to the region upstream of the zip-1 gene coding sequence, which contains annotated $5^{\prime}$ untranslated regions (UTRs) for several $z i p-1$ isoforms, as well as to downstream sequences that contain the zip-1 $3^{\prime}$ UTR (Supplementary Fig. 9). This finding indicates that $z i p-1$ is not required to induce its own transcription, but rather a distinct transcription factor is involved in upregulation of $z i p-1$ mRNA expression.

To obtain insight into other biological processes and cellular structures that may be related to zip-1, we performed analysis with the WormCat program, specifically designed for analysis of
C. elegans genomics data ${ }^{26}$. We separately analyzed 64 genes from the early timepoint and 888 genes from the later timepoint that were upregulated in wild-type animals but not zip-1 mutants. The only significantly overrepresented category of upregulated genes at $30 \mathrm{~min}$ was the stress response category (Fig. $4 \mathrm{~d}$, Supplementary Table 1). Analysis of the genes upregulated at $4 \mathrm{~h}$ revealed a significant overrepresentation of genes implicated in mRNA function, transcription, nuclear pore, signaling, development, cytoskeleton, proteolysis, and DNA.

Finally, we analyzed and classified 80 canonical IPR genes ${ }^{13}$ based on their expression levels in our RNA-seq datasets, to place them into different categories based on their dependence on zip1. Here we found that 23 IPR genes (including pals-5) were upregulated in wild-type animals but not zip-1 mutants $30 \mathrm{~min}$ after bortezomib treatment, but became upregulated in both genetic backgrounds at $4 \mathrm{~h}$ (Fig. 4e). Therefore, these genes are 
"Early zip-1-dependent" IPR genes. Notably, 11 pals genes belong to this category. Another seven IPR genes (including skr-5) were not upregulated in zip-1(jy13) mutants at either timepoint analyzed, but were upregulated in wild-type at $4 \mathrm{~h}$, and we classified these genes as "Late zip-1-dependent" IPR genes. Therefore, overall, 30 IPR genes appeared to be zip-1-dependent, when including both timepoints. 42 canonical IPR genes were upregulated in both genetic backgrounds, and we classified them as "zip-1-independent" IPR genes. Because some of these genes were not upregulated at the first timepoint, we further divided this category of genes into class A that showed upregulation after 30 min bortezomib treatment (including F26F2.1), and class B that showed upregulation only after $4 \mathrm{~h}$ of bortezomib treatment. Of note, eight canonical IPR genes did not show significant upregulation after bortezomib treatment, so we did not classify them in any category. These include histone genes, which previous studies had shown to be regulated by pals-22/pals-25 and $N$. parisii infection (and thus qualify as IPR genes), but not to be induced by bortezomib treatment ${ }^{11,13}$. In conclusion, our RNAseq results demonstrate that $z i p-1$ controls RNA expression of 30 out of 80 IPR genes, and reveal that IPR genes can be placed into three separate classes based on their regulation by zip-1.

ZIP-1 is expressed in the intestine and is at least partially required in this tissue to regulate pals-5 gene expression. To examine where ZIP-1 is expressed, we tagged the zip-1 endogenous genomic locus with $g f p$ immediately before the stop codon using CRISPR/Cas9-mediated gene editing. Here we found that ZIP-1::GFP endogenous expression was not detectable in unstressed animals. Because zip-1 mRNA is induced by bortezomib, and bortezomib blocks protein degradation, we investigated whether ZIP-1::GFP was visible after bortezomib treatment. Here we found that ZIP-1::GFP expression was induced, with strongest expression found in intestinal nuclei (Fig. 5a). Nuclear expression was also identified in the epidermis (Fig. 5b). Specifically, 98\% (59/60) of animals showed ZIP-1::GFP expression in intestinal nuclei after $4 \mathrm{~h}$ bortezomib treatment, while $88 \%$ (53/ 60) showed expression in epidermal nuclei after $4 \mathrm{~h}$ bortezomib treatment. In contrast, no GFP signal was observed in wild-type animals treated with bortezomib, or in zip-1::gfp mutants or wildtype animals treated with DMSO control (60 analyzed animals for each condition).

Next we examined ZIP-1::GFP expression upon intracellular infection. First, we infected zip-1::gfp animals with $N$. parisii, then stained with a FISH probe to label parasite cells, and quantified the percentage of infected animals displaying ZIP-1::GFP nuclear expression. Here we found that $73.33 \%(44 / 60)$ of animals with visible parasite cells had ZIP-1::GFP expression, in contrast to $0 \%$ $(0 / 60)$ of uninfected animals that had ZIP-1::GFP expression (Fig. 5c). We did note that uninfected intestinal cells found adjacent to $N$. parisii-infected cells sometimes displayed ZIP$1:: G F P$ expression, suggesting there may be cell-to-cell signaling from infected to uninfected cells to induce ZIP-1::GFP expression. However, we cannot eliminate the possibility that in these cases the 'uninfected' cells actually had pathogen present, but it was not visible.

We next examined ZIP-1::GFP expression in animals infected with Orsay virus. Here, we found that $100 \%$ (60/60) virus infected animals had ZIP-1::GFP expression, in contrast to $0 \%(0 / 60)$ of uninfected animals (Fig. 5d). Similar to infection with N. parisii, we found evidence there may be cell-to-cell signaling upon Orsay virus infection, as uninfected cells adjacent to viral-infected cells sometimes displayed ZIP-1::GFP expression. Importantly, because virus is sensed by the DRH-1 receptor to activate the IPR, our finding that viral infection induces ZIP-1::GFP expression enabled us to determine whether DRH-1 acts upstream of ZIP-1. Here we found that viral infection no longer induced ZIP-1::GFP in $d r h-1$ mutants ( $0 \%$ or $0 / 60$ animals) (Fig. 5e), suggesting that the DRH-1 receptor acts upstream of the ZIP-1 transcription factor.

To determine the tissue in which zip-1 acts to regulate pals-5 induction, we performed tissue-specific downregulation of zip-1 using RNAi, and measured the levels of pals-5 mRNA following $30 \mathrm{~min}$ bortezomib treatment. First, we used rde-1 loss-offunction mutation strains, which have a $r d e-1$ rescuing construct expressed specifically in either the intestine or in the epidermis, which leads to enrichment of RNAi in these tissues. Here we observed that zip-1 RNAi in the intestinal-specific RNAi strain caused a block in pals-5 induction, similar to zip-1(RNAi) in wildtype animals (Fig. 5f). In contrast, pals-5 induction was less compromised by zip-1(RNAi) in the epidermal-specific RNAi strain. However, because intestinal expression of $r d e-1$ allows generation of secondary siRNAs that can spread to other tissues and silence gene expression there, $r d e-1$ strains can be somewhat leaky. Therefore, we analyzed the intestinal requirement for zip-1 in a separate tissue-specific RNAi strain, where the sid-1 transport channel is specifically expressed in the intestine in a sid-1 mutant background 27,28 . This strain does not suffer from the problem of leakiness seen in the $r d e-1$ rescue strains ${ }^{28,29}$. However, we did note that this strain suffers from the opposite problem: they appear to be somewhat resistant to RNAi. To quantify this effect, we treated the intestinally rescued sid-1 strain with dsRNA against act-5, which is an actin isoform expressed in the intestine, but not in the epidermis. Because act-5 is essential for development, we could use act-5(RNAi) to determine the efficacy of RNAi in this intestinally rescued sid- 1 strain $^{30}$. Here we found that act-5(RNAi) caused less severe effects on size in the intestinally rescued sid-1 strain compared to wild-type animals (Supplementary Fig. 10a, b). Despite being partially resistant to RNAi, we did find that zip-1(RNAi) in this strain caused a significant reduction in pals-5 mRNA induction upon bortezomib treatment compared to vector control (Supplementary Fig. 10c). Taken together, our data suggest that zip-1 is highly expressed in the intestinal nuclei following IPR triggers, and that $z i p-1$ is at least partially required in the intestine for induction of pals5 mRNA.

zip-1 regulates resistance to natural intracellular pathogens. Because increased IPR gene expression is correlated with increased resistance to intracellular infection ${ }^{10,13}$, we investigated the role of zip-1 in resistance to intracellular pathogens. First, we investigated Orsay virus. Here, we infected L4 animals and found that zip-1 mutants had higher viral load compared to wild-type animals, as assessed by qRT-PCR (Fig. 6a). Similarly, we found upon infection of L1 animals and measuring viral load with FISH staining that zip-1 mutants had a trend toward higher infection rate than wild-type animals (Fig. 6b). We also investigated whether zip-1 might have a greater effect on viral load in a mutant background where IPR genes are constitutively expressed. Indeed, we found a more pronounced role for $z i p-1$ after viral infection of pnp-1 mutants, which have constitutive expression of IPR genes, including pals-5 (Fig. 6b) ${ }^{15}$. Of note, our qRT-PCR analysis of pnp-1(jy90) animals showed that elevated pals-5 mRNA levels depend on zip-1, suggesting that the IPR genes upregulated by $z i p-1$ promote resistance against viral infection (Supplementary Fig. 11). Similar to what we observed after bortezomib treatment, expression of highly induced IPR genes F26F2.1, F26F2.3, and F26F2.4 in a $p n p-1$ mutant background did not require zip-1. This finding suggests that $z i p$-1-dependent IPR genes may play a more important role in Orsay virus resistance than other IPR genes. 

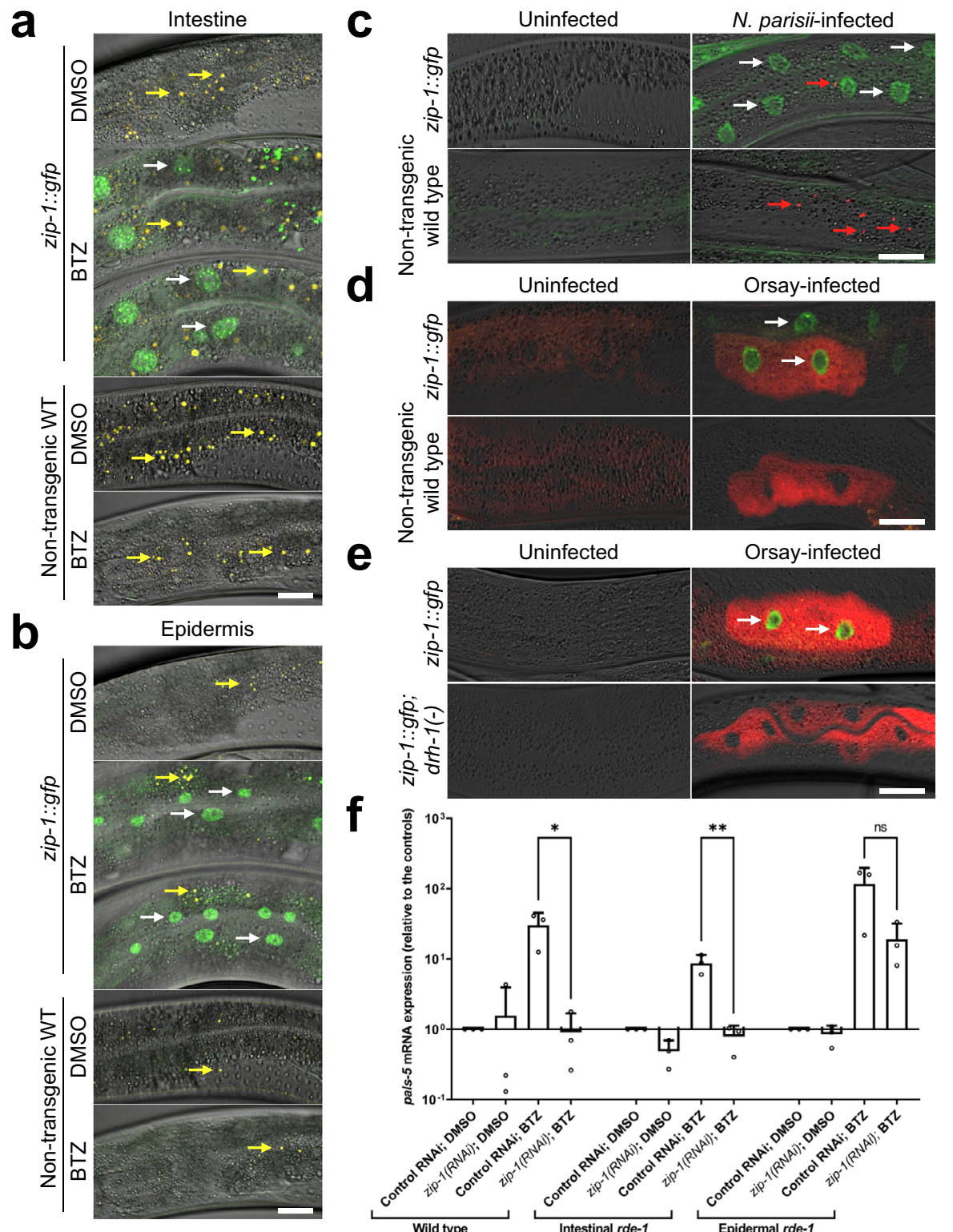

e
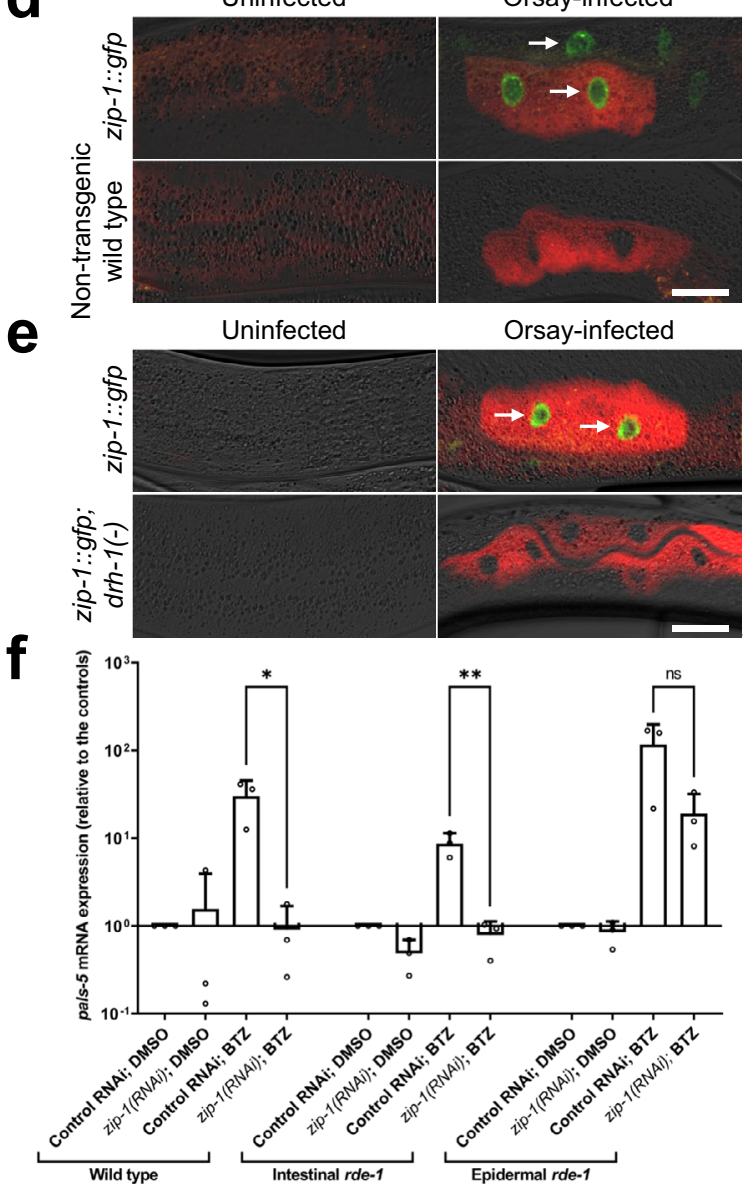

Fig. 5 zip-1 acts in the intestine to regulate pals-5 mRNA levels. a, b ZIP-1::GFP is expressed in intestinal (a) and epidermal nuclei (b) $4 \mathrm{~h}$ after bortezomib treatment. No expression was observed in animals exposed to DMSO control, or in the non-transgenic control strain N2. Composite images consist of merged fluorescent (GFP and autofluorescence) and DIC channels. Yellow signal in the composite images depicts autofluorescence from gut granules. White arrows indicate representative intestinal nuclei expressing ZIP-1::GFP; yellow arrows indicate autofluorescence. Scale bar $=20 \mu \mathrm{m}$. c-e $\mathrm{N}$. parisii (c) and Orsay virus infection (d, e) induce ZIP-1::GFP expression in intestinal nuclei. Fluorescent and DIC images were merged; green represents ZIP-1::GFP; fluorescence from pathogen-specific FISH probes is shown in red. White arrows indicate representative intestinal nuclei expressing ZIP-1::GFP (c-e); red arrows indicate $N$. parisii sporoplasms $(\mathbf{c})$. Scale bar $=30 \mu \mathrm{m}$. $\mathbf{f}$ Intestine-specific zip-1(RNAi) prevents pals-5 mRNA induction. qRT-PCR measurements of pals-5 levels at the 30 min timepoint of bortezomib (BTZ) or DMSO treatments. The results are shown as fold change in gene expression relative to DMSO diluent control. Three independent experimental replicates were analyzed; the values for each replicate are indicated with circles. Bar heights indicate mean values and error bars extend above. Error bars represent standard deviations. A one-tailed $t$-test was used to calculate $p$-values; ${ }^{\star \star} p<0.01 ;{ }^{\star} 0.01<p<0.05$; ns indicates nonsignificant difference $(p>0.05)$. $p$-values for WT control RNAi BTZ vs zip-1(RNAi) BTZ $p=0.0170$; intestinal rde-1 control RNAi BTZ vs zip$1(R N A i)$ BTZ $p=0.0092$; epidermal rde-1 control RNAi BTZ vs zip-1(RNAi) BTZ $p=0.0614$. Source data are provided as a Source Data file.

Next, we examined a role for zip-1 in resistance to $N$. parisii infection by measuring pathogen load. Here we did not see an effect of $z i p-1$ in a wild-type background either at $3 \mathrm{hpi}$ or at 30 hpi (Fig. 6c, d). However, at both timepoints, we found that loss of $z i p-1$ significantly suppressed the increased pathogen resistance (i.e., lower pathogen load) of pnp-1 mutants (Fig. 6c, d). Therefore, these experiments indicate that wild-type zip-1 promotes resistance to $N$. parisii infection in a background where IPR genes are induced prior to infection. To further analyze the role of $z i p-1$ in response to $N$. parisii infection, we also performed killing assays in which we analyzed survival of animals following infection. Consistent with published data, we found that pnp-1 mutants survive longer than wild-type animals when infected with $N$. parisii, but do not survive longer than wild-type animals in the absence of infection (Fig. 6e, f). Importantly, we found that $z i p-1$ mutations decrease survival both in a pnp-1 mutant background, as well as in a wild-type background. Therefore, wild-type zip-1 promotes survival against $N$. parisii 
a

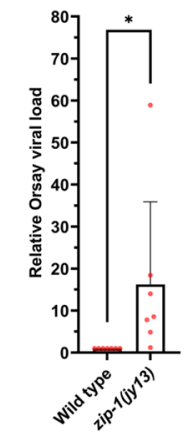

e

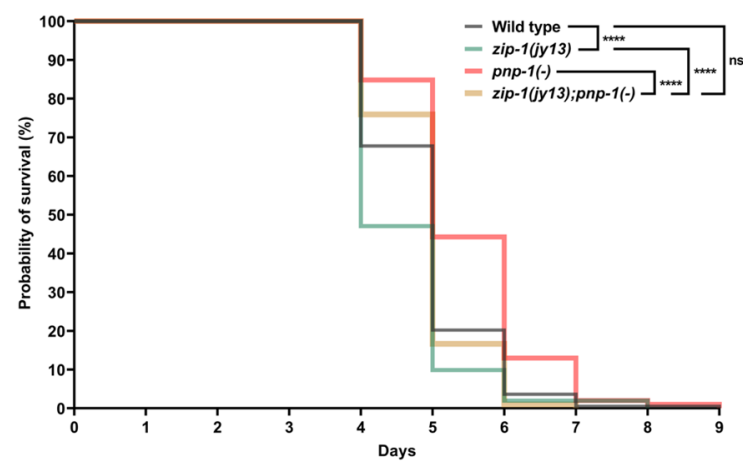

C

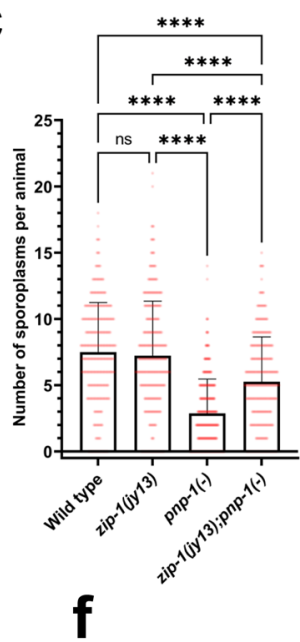

d

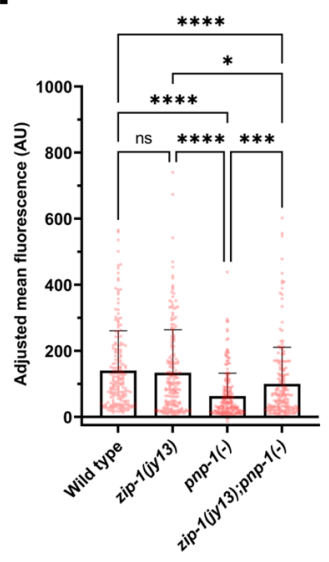

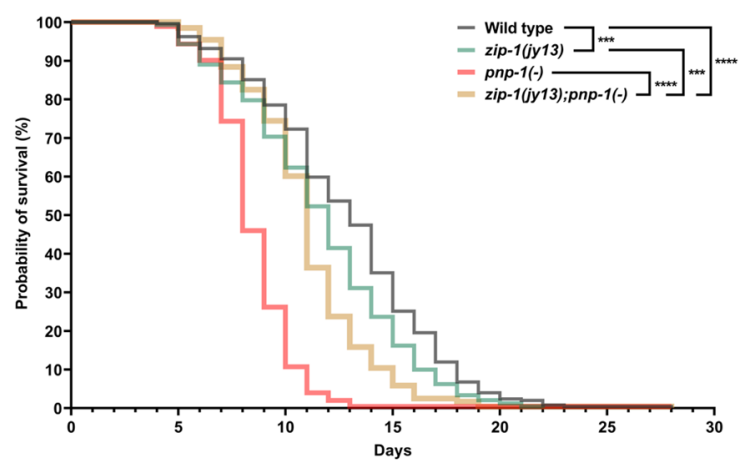

Fig. 6 zip-1 promotes resistance to intracellular pathogens. a qRT-PCR analysis of Orsay virus RNA1 levels in control and zip-1(jy13) mutant animals. Animals were infected at L4 stage and collected at $24 \mathrm{hpi}$. Seven experimental replicates were analyzed, each consisting of two biological replicates assayed in technical duplicates. b Fraction of animals infected with Orsay virus in control, zip-1(jy13), pnp-1(jy90) and zip-1(jy13); pnp-1(jy90) backgrounds at $12 \mathrm{hpi}$. Animals were infected at L1 stage. 900 animals per strain were scored based on the presence or absence of the Orsay virus RNA1-specific FISH probe fluorescence (three experimental replicates, 300 animals per replicate). The infection rate of the control strain was set to one. c N. parisii pathogen load quantified at $3 \mathrm{hpi}$ as number of sporoplasms per animal; $300 \mathrm{~L} 1$ animals were analyzed per strain in three experimental replicates. $\mathbf{d}$ Quantification of N. parisii-specific mean FISH fluorescence signal normalized to body area excluding pharynx. Animals were infected at L1 stage and analyzed at 30 hpi; 200 animals were analyzed per strain (four experimental replicates, 50 animals per replicate). The head region was excluded from the analysis because of the expression of the red coinjection marker myo-2p::mCherry. AU arbitrary units. a-d Bar heights indicate mean values and error bars extend above. Error bars represent standard deviations. e Survival of wild-type, zip-1(jy13), pnp-1(jy90) and zip-1(jy13); pnp-1(jy90) animals following N. parisii infection. Animals were exposed to N. parisii spores for $66 \mathrm{~h}$ from L1 stage, and then transferred daily to non-infectious plates and scored for viability. $\mathbf{f}$ Longevity analysis of strains used in the infection assays. e, f Animals were incubated at $25^{\circ} \mathrm{C}$. Data from 3 experimental replicates are shown in a single graph. Percentage of alive animals is indicated on $y$-axis for each day of analysis ( $x$-axis). a-f Statistical analyses were performed using a one-tailed $t$-test (a), an ordinary one-way ANOVA (b), a Kruskal-Wallis (c, d), and a log-rank (Mantel-Cox) test $(\mathbf{e}, \mathbf{f})$ to calculate $p$-values; ${ }^{\star \star \star \star} p<0.0001 ;{ }^{* \star \star} p<0.001$; ${ }^{* \star} p<0.01 ;{ }^{\star} 0.01<p<0.05$; ns indicates nonsignificant difference $(p>0.05)$. $p$-value in a for WT vs zip-1(jy13) $p=0.0312$. $p$-values in $\mathbf{b}$ for WT vs zip-1(jy13) $p=0.5813$; WT vs pnp1(jy90) $p=0.0079$; WT vs zip-1(jy13); pnp-1(jy90) $p>0.9999 ;$ zip-1(jy13) vs pnp-1(jy90) $p=0.0017$; zip-1(jy13) vs zip-1(jy13); pnp-1(jy90) $p=0.6145 ; p n p-$ 1(jy90) vs zip-1(jy13); pnp-1(jy90) $p=0.0073$. $p$-values in c for WT vs zip-1(jy13) $p>0.9999$; for all other comparisons $p<0.0001$. $p$-values in $\mathbf{d}$ for WT vs zip-1(jy13) $p=0.4025$; WT vs pnp-1(jy90), WT vs zip-1(jy13); pnp-1(jy90) and zip-1(jy13) vs pnp-1(jy90) p<0.0001; zip-1(jy13) vs zip-1(jy13); pnp-1(jy90) $p=0.0312 ;$ pnp-1(jy90) vs zip-1(jy13); pnp-1(jy90) $p=0.0010$. $p$-values in e for WT vs zip-1(jy13), zip-1(jy13) vs zip-1(jy13); pnp-1(jy90) and pnp-1(jy90) vs zip1(jy13); pnp-1(jy90) $p<0.0001$; WT vs zip-1(jy13); pnp-1(jy90) $p=0.7781$. $p$-values in f for WT vs zip-1(jy13) $p=0.0005$; WT vs zip-1(jy13); pnp-1(jy90) and pnp-1(jy90) vs zip-1(jy13); pnp-1(jy90) $p<0.0001 ;$ zip-1(jy13) vs zip-1(jy13); pnp-1(jy90) $p=0.0009$. All strains are in a pals-5p::gfp background. Source data are provided as a Source Data file.

infection. Because infections were performed by feeding pathogens to animals, it was possible that differences in food intake and elimination were responsible for any differences seen in pathogen load. Therefore, we measured accumulation of fluorescent beads in all tested strains and we did not find any significant differences between zip-1 mutants and control animals (Supplementary Fig. 12). In conclusion, the increased pathogen load in $z i p-1$ mutants is unlikely to be due to differences in the exposure of intestinal cells to pathogen in these mutants.

Other phenotypes in pnp-1 mutants include higher sensitivity to heat shock and slightly slower growth rate ${ }^{15}$. We tested if either of these phenotypes are zip-1-dependent. First, we found that zip1(jy13) animals had a similar survival rate after heat shock compared to the control strain (Supplementary Fig. 13a). Similarly, we found that loss of zip-1 in a pnp-1(jy90) mutant background did not significantly suppress the higher lethality observed in pnp-1(jy90) single mutants, suggesting that ZIP-1 does not play a crucial role in thermotolerance regulation. Finally, we analyzed if zip-1(jy13) mutants, which show a wild-type growth rate, can suppress the mild growth delay caused by a $p n p$ 1(jy90) mutation. Here, growth was assayed based on the body length measurements $44 \mathrm{~h}$ after plating synchronized L1 animals, 
and we found that $z i p-1(j y 13) ; p n p-1(j y 90)$ animals were still significantly smaller than control animals and zip-1(jy13) single mutants (Supplementary Fig. 13b). Therefore, zip-1 does not appear to be important for these noninfection-related phenotypes of $p n p-1$ mutants. Instead, it seems that $z i p-1$ specifically plays a role in regulating immunity-related IPR genes.

\section{Discussion}

Most studies of antiviral immunity in invertebrates have focused on antiviral RNAi, and less is known about transcriptional responses to intracellular infection in either of the two major invertebrate model systems, Drosophila melanogaster or C. elegans $^{31-33}$. The IPR in C. elegans is a common transcriptional response that is induced independently by both virus and microsporidia infection, as well as by specific physiological perturbations such as proteotoxic stress ${ }^{11-13}$. Previous studies had shown that the STAT-related transcription factor sta-1 was a repressor of IPR genes ${ }^{34}$, but the activating transcription factor for the IPR was not known. Here, we show that the previously uncharacterized, predicted bZIP transcription factor ZIP-1 functions downstream of all known IPR triggers to induce a subset of IPR genes (Fig. 7). Importantly, we show that $z i p-1$ plays a role in immunity against infection by both the Orsay virus and microsporidia. Therefore, zip-1 appears to be the first transcription factor shown to promote an inducible defense response against intracellular intestinal pathogens in C. elegans.

ZIP-1 adds to the growing list of bZIP transcription factors involved in C. elegans immunity. The bZIP transcription factor family is expanded in C. elegans compared to other organisms ${ }^{35}$ and several members of this family have been previously implicated in defense against the extracellular bacterial pathogens of the intestine. For example, the central pathway in defense against bacterial pathogens like Pseudomonas aeruginosa is the p38 MAPK pathway, which leads to activation of the ATF-7 bZIP transcription factor, as well as the bZIP-related transcription factor SKN-1 in response to reactive oxygen species generated upon infection ${ }^{36,37}$. The bZIP proteins ZIP-2 and CEBP-2 control induction of several p38-independent genes induced by
$P$. aeruginosa, in response to the $P$. aeruginosa translationblocking Exotoxin $\mathrm{A}^{38-41}$. Under certain infection conditions, ZIP-2 and CEBP-2 act with two other bZIP transcription factors, ZIP-4 and CEBP-1, to control induction of the ethanol and stress response element network upon $P$. aeruginosa infection, likely in response to mitochondrial damage ${ }^{42}$. Furthermore, the bZIP proteins ATFS-1 and ZIP-3 have been shown to play antagonistic roles in activation of mitochondrial unfolded protein response upon damage caused by $P$. aeruginosa infection ${ }^{43,44}$.

In addition to the bZIP transcription factors mentioned above, several other classes of transcription factors play roles in C. elegans defense, including FOXO, GATA, HSF, HLH, and NHR transcription factor family members ${ }^{45-50}$. Moreover, several members of C. elegans Myc family of transcription factors have been shown to be the regulators of microsporidia growth and development ${ }^{19}$. What is the logic to having so many transcription factors involved in immunity in C. elegans? For comparison, only one bZIP transcription factor, CrebA, has recently been shown to play a role in D. melanogaster tolerance to bacterial pathogen ${ }^{51}$. Also, a single STAT transcription factor, a component of JAK/ STAT pathway, has been shown to play a downstream role in antiviral immunity, although this factor is not thought to be the first responder to viral infection ${ }^{52,53}$. The majority of studies in $D$. melanogaster indicate that the NF-kB transcription factors Dif, Dorsal and Relish are the major transcription factors to induce immune genes upon bacterial and fungal infections, and they also play a role in antiviral immunity ${ }^{53-55}$. A large percentage of immune genes in humans are also controlled by NF-kB transcription factors upon induction by bacterial infection, and by IRF3/7 upon viral infection, working together with NF-kB ${ }^{56-59}$. Interestingly, NF-kB was lost in the evolutionary lineage that gave rise to $C$. elegans, so perhaps several other transcription factors fill that gap to induce defense ${ }^{14}$. Or perhaps, this diverse list of immune-related transcription factors is a result of C. elegans apparently lacking professional immune cells like macrophages or hemocytes, which play key roles in mammalian and D. melanogaster defense, respectively ${ }^{60,61}$. For this reason, studies in $C$. elegans have focused on nonprofessional immune cells like

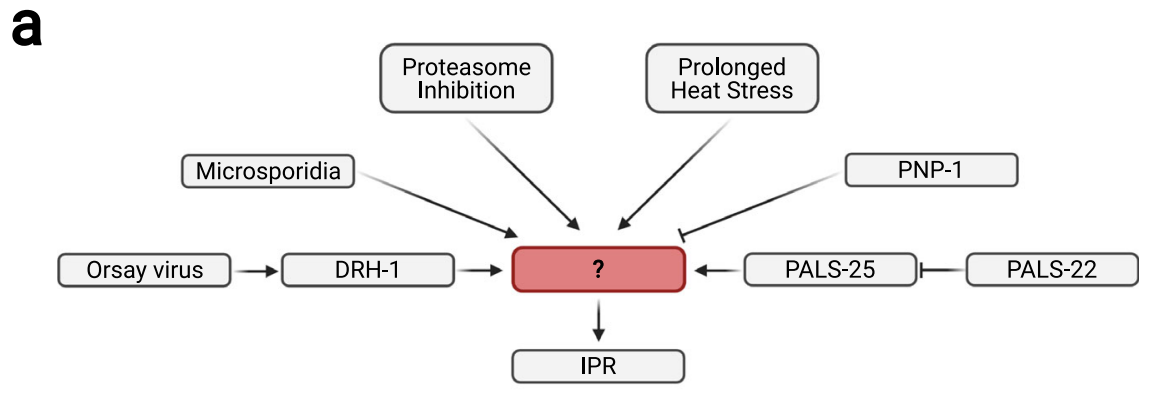

b

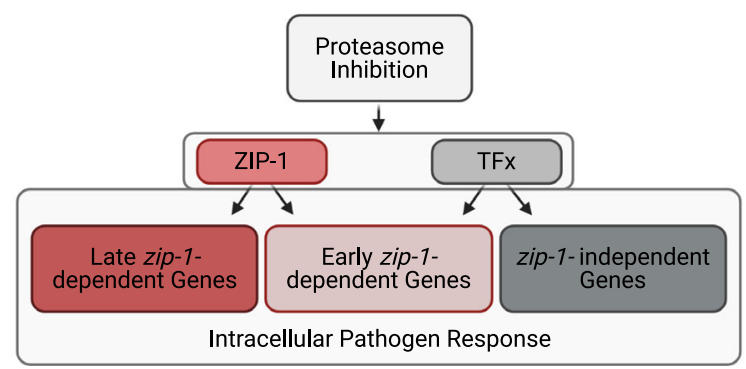

Fig. 7 Model of IPR gene regulation. a All known IPR-activating pathways require ZIP-1 for induction of the pals-5p::GFP reporter. b IPR genes can be divided into three categories: early zip-1-dependent, late zip-1-dependent and zip-1-independent genes. Unknown transcription factor or factors (TFX) regulate expression of early zip-1-dependent genes at later timepoint, as well as transcription of zip-1-independent genes. Created with BioRender.com. 
epithelial cells ${ }^{62-64}$, which are less well studied in mammalian research compared to professional immune cells like macrophages. If more mammalian and D. melanogaster studies screened for transcription factors acting in epithelial cells, the lists might grow longer there as well.

Although our study indicates that ZIP-1 plays an important role in defense against intracellular infection, it almost certainly is not the only transcription factor with such a role. Our qRT-PCR and RNA-seq analyses demonstrated that many genes induced as part of the IPR do not require ZIP-1 for their induction, while some require ZIP-1 only for early induction, but not late induction. Future studies with screens for transcription factors that mediate induction of $z i p$-1-independent genes should enable a more complete assessment of the immune response to intracellular infection in C. elegans.

In this study we demonstrate that ZIP-1 protein expression can be triggered by several different signaling pathways that induce the IPR, including proteasome inhibition and viral infection acting through the DRH-1 receptor ${ }^{10}$. While zip-1 itself appears to be transcriptionally and translationally induced by infection, we believe that ZIP-1 is the immediate transcription factor that activates IPR gene expression downstream of various triggers, based on the following reasoning. Specifically, zip-1 is required for IPR gene induction only $30 \mathrm{~min}$ after activation by bortezomib, which is likely too short a time for a separate transcription factor to activate $z i p-1$ transcription and translation, which would then induce IPR gene expression. There is still much to be learned about how various triggers activate ZIP-1, which then upregulates IPR gene expression, although a likely ligand and receptor pair have been identified for the Orsay virus, where viral RNA replication products appear to be detected by the RIG-I-like receptor DRH- $1^{10}$. As mentioned earlier, C. elegans lacks the downstream factors that mediate viral/RIG-I signaling in mammals, such as IRF3/7 and interferon. Therefore, we propose that ZIP-1 and the IPR may play an analogous role to IRF3/7 and interferon in $C$. elegans defense against intracellular infection in intestinal epithelial cells. Further analysis should shed light on how the evolutionarily ancient RIG-I-like receptor family is rewired in C. elegans to enable activation of ZIP-1 and downstream defense against intracellular infection.

\section{Methods}

Worm maintenance. Worms were grown on Nematode Growth Media (NGM) plates seeded with Streptomycin-resistant E. coli OP50-1 bacteria at $20^{\circ} \mathrm{C}$, unless stated otherwise. Strains used in this study are listed in the Supplementary Table 2.

RNAi screens. RNAi screens were performed using the feeding method in liquid medium. Gravid adults were bleached following a standard protocol ${ }^{65}$, and isolated eggs were incubated in M9 medium overnight to hatch into starved L1's unless stated otherwise. In particular, for the screen in the pals-22(jy3) mutant background, eggs isolated from bleached gravid adults were put on OP50 seeded NGM plates and incubated at $20^{\circ} \mathrm{C}$ for $48 \mathrm{~h}$. Subsequently, animals were washed off the plates with S-basal medium and 150 animals were transferred into wells of 96-well plates. Overnight cultures of RNAi HT115 bacterial strains were supplemented with $2.2 \mathrm{mM}$ isopropyl $\beta$-d-1-thiogalactopyranoside (IPTG) and $1 \mathrm{mM}$ carbenicillin, and added to the wells with worms. Control RNAi experiments were carried out using L4440 (negative control vector) and $g f p(R N A i)$ (positive control). Following incubation at $20^{\circ} \mathrm{C}$ for $48 \mathrm{~h}$, animals were collected and analyzed on the COPAS Biosort machine (Union Biometrica). PALS-5::GFP signal and the time-offlight (TOF, as a measure of length) were quantified, and average values for fluorescence/body length were calculated for each animal. In Supplementary Data 1 we also list normalized TOF values as a proxy for body length, which indicates that an RNAi clone like lin-26 not only had low GFP/TOF, but also had low TOF, indicating small size and thus potentially poor overall health, and thus was not pursued as a hit. The transcription factor RNAi library is split among five 96-well plates. Three of these plates were tested in two experimental replicates and two of these plates were tested in one experimental replicate.

For the screen in which chronic heat stress was used to induce the IPR, synchronized populations of $150 \mathrm{~L} 1$ animals carrying the jyIs8[pals-5p::gfp] transgene were transferred to S-basal medium in 96-well plates. The wells were supplemented with overnight RNAi bacterial cultures, as previously described for
RNAi screen in pals-22(jy3) mutant background. Animals were incubated in the shaker at $20^{\circ} \mathrm{C}$ for $48 \mathrm{~h}$, and then subjected to chronic heat stress at $30^{\circ} \mathrm{C}$ for $18 \mathrm{~h}$. Subsequently, pals-5p::GFP expression was measured and standardized to the worm length using TOF measurements on the COPAS Biosort machine. Three independent experimental replicates were performed.

RNAi assays on plates. RNA interference assays were performed using the feeding method. Overnight cultures of HT115 E. coli were plated on RNAi plates (NGM plates supplemented with $5 \mathrm{mM}$ IPTG and $1 \mathrm{mM}$ carbenicillin) and incubated at room temperature for 3 days. Synchronized L1 animals were transferred to these plates and incubated at $20^{\circ} \mathrm{C}$. Following $48 \mathrm{~h}$ incubation, specific phenotypes of animals were analyzed (pals-5p::GFP expression after exposure to zip-1 RNAi; developmental defects after act-5 RNAi treatment). Control RNAi experiments were carried out using a vector plasmid L4440.

CRISPR/Cas9-mediated deletions of zip-1 and pals-5. Deletions of zip-1 and pals-5 were carried out using the co-CRISPR method with preassembled ribonucleoproteins ${ }^{66,67}$. Cas9-NLS protein $(27 \mu \mathrm{M}$ final concentration) was ordered from QB3 Berkeley; sgRNA components and DNA primers were obtained from Integrated DNA Technologies (IDT).

The following crRNA sequences were used to target zip-1 gene: acacaggcatctggggaccc (for generating the jy13 allele), tcagcttgtgctgggcgttg (for generating the $j y 14$ allele), agcaatttgagccaagctga (for generating both jy13 and jy14 alleles). PCR screenings were performed using the primers 1-4 listed in Supplementary Table 3. Deletion-positive lines were backcrossed three times to the $\mathrm{N} 2$ strain before they were used in experiments. The jy13 allele is an 8241 base pair long deletion, starting 172 nucleotides upstream of the zip-1 start codon and ending at the last nucleotide before the stop codon (C8069). jy14 allele is a 4108 base pair long deletion, starting at nucleotide G3962 and ending at the last nucleotide before the stop codon (C8069).

The following crRNA sequences were used to target the pals-5 gene: aaatactcgaagcaattcag and aaaacgaatagaaaatggga. PCR screenings were performed using primers 10 and 11 from the Supplementary Table 3. Deletion-positive lines were backcrossed three times to the N2 strain before they were used in experiments. jy133 allele is a 1706 base pair long deletion, starting 128 nucleotides upstream of the pals-5 start codon and ending at the 108th nucleotide after the stop codon.

Orsay virus infections. Orsay virus isolate was prepared as previously described ${ }^{11}$ For pals-5p::GFP expression analysis and FISH staining for infection level quantification, L1 animals were exposed to a mixture of OP50-1 bacteria and Orsay virus for $12 \mathrm{~h}$ at $20^{\circ} \mathrm{C}$, whereas animals used for ZIP-1::GFP analysis were infected with a high dose of virus for $9 \mathrm{~h}$ at $20^{\circ} \mathrm{C}$. pals-5p::GFP reporter expression was analyzed in animals that were anesthetized with $10 \mathrm{mM}$ levamisole. For FISH analysis, animals were collected and fixed in $4 \%$ paraformaldehyde for $15-45 \mathrm{~min}$ depending on the assay. Fixed worms were stained at $46^{\circ} \mathrm{C}$ overnight using FISH probes conjugated to the red Cal Fluor 610 fluorophore, targeting Orsay virus RNA1. GFP imaging and FISH analysis were performed using Zeiss AxioImager M1 compound microscope. For qRT-PCR analyses, synchronized L4 animals were exposed to a mixture of OP50-1 bacteria and Orsay virus for $24 \mathrm{~h}$ at $20^{\circ} \mathrm{C}$. RNA isolation and qRT-PCR analysis were performed as described below. ZIP-1::GFP expression was analyzed and imaged on a Zeiss LSM700 confocal microscope run by ZEN2010 software.

Microsporidia infections. $N$. parisii spores were prepared as previously described ${ }^{63}$. Spores were mixed with food and L1 synchronized animals (a dose of 8 million spores per plate was used for ZIP-1::GFP expression analyses, a dose of 0.5 million spores per plate was in all other assays). Animals were incubated at $25^{\circ} \mathrm{C}$ for $3 \mathrm{~h}$ (for sporoplasm counting and ZIP-1::GFP analysis), $24 \mathrm{~h}$ (for qRTPCR analysis of IPR gene expression) or $30 \mathrm{~h}$ (for pathogen load analysis). For pals$5 \mathrm{p}:$ GFP expression analysis, animals were anesthetized with $10 \mu \mathrm{M}$ levamisole and imaged using Zeiss AxioImager M1 compound microscope. For FISH analysis, animals were collected and fixed in 4\% paraformaldehyde for 15-45 min depending on the assay. Fixed worms were stained at $46^{\circ} \mathrm{C}$ for $6 \mathrm{~h}$ (for ZIP-1::GFP analysis) or overnight (for pathogen load analyses) using FISH probes conjugated to the red $\mathrm{Ca}$ Fluor 610 fluorophore, targeting ribosomal RNA. 3 hpi samples were analyzed using Zeiss AxioImager M1 compound microscope; 30 hpi samples were imaged using ImageXpress automated imaging system Nano imager (Molecular Devices, LLC), and fluorescence levels were analyzed using FIJI program. ZIP-1::GFP expression was analyzed and imaged on a Zeiss LSM700 confocal microscope run by ZEN2010 software.

Bortezomib treatments. Proteasome inhibition was performed using bortezomib (Selleckchem, catalog number S1013) as previously described ${ }^{18,22}$. Synchronized L1 animals were plated on $10 \mathrm{~cm}$ (for RNA extraction) or $6 \mathrm{~cm}$ NGM plates (for phenotypic analyses and transgene expression measurements), and grown for $44 \mathrm{~h}$ or $48 \mathrm{~h}$ at $20^{\circ} \mathrm{C}$ depending on the assay. $10 \mathrm{mM}$ stock solution of bortezomib in DMSO was added to reach a final concentration of $20 \mu \mathrm{M}$ per plate. The same volume of DMSO was added to the control plates. Plates were dried and worms 
incubated for $30 \mathrm{~min}, 4,21$, or $25 \mathrm{~h}$ at $20^{\circ} \mathrm{C}$. Imaging was performed using Zeiss AxioImager M1 compound microscope or ImageXpress automated imaging system Nano imager (Molecular Devices, LLC), and analyzed using FIJI program. For RNA extraction, animals were washed off the plates using M9, washed with M9 and collected in TRI reagent (Molecular Research Center, Inc.). ZIP-1::GFP expression was analyzed and imaged on a Zeiss LSM700 confocal microscope run by ZEN2010 software.

Fluorescence measurements. Fluorescence measurements shown in Figs. 1a, b and $2 \mathrm{~d}$ were performed using the COPAS Biosort machine (Union Biometrica). The fluorescent signal was normalized to TOF, as a proxy for worm length. Fluorescence measurements shown in Figs. 2f and 6d and Supplementary Fig. 12 were performed by imaging animals using ImageXpress automated imaging system Nano imager (Molecular Devices, LLC), followed by image analysis in FIJI. Mean gray value (as a ratio of integrated density and analyzed area) was measured for each animal and normalized to the background fluorescence.

Bioluminescence measurements. Synchronized L1 animals were grown at $20^{\circ} \mathrm{C}$ for $44 \mathrm{~h}$ and then treated with bortezomib or DMSO for $4 \mathrm{~h}$. Sample preparation and nanoluciferase bioluminescence measurements were performed as previously described $^{22}$. In brief, animals were collected and disrupted using silicon carbide beads in lysis buffer (50 mM HEPES pH 7.4, $1 \mathrm{mM}$ EGTA, $1 \mathrm{mM} \mathrm{MgCl}_{2}, 100 \mathrm{mM}$ $\mathrm{KCl}, 10 \%$ glycerol, $0.05 \%$ NP40, $0.5 \mathrm{mM}$ DTT, protease inhibitor cOmplete (Sigma, catalog number 11836170001)). The lysates were centrifuged and the supernatants were collected and stored at $-80^{\circ} \mathrm{C}$ until bioluminescence was measured. NanoGlo Luciferase Assay System reagent (Promega, catalog number N1110) was added to the worm lysate supernatant before analysis, and incubated at room temperature for $10 \mathrm{~min}$. Analysis was performed on a NOVOstar plate reader. The results were obtained and normalized to blank controls using Mars data analysis software (BMG LABTECH).

smFISH analysis. smFISH experiments were performed as previously described ${ }^{12}$ In brief, L4 animals were treated with bortezomib or DMSO for $4 \mathrm{~h}$ at $20^{\circ} \mathrm{C}$. Animals were washed off the plates, fixed in $4 \%$ paraformaldehyde in phosphatebuffered saline $+0.1 \%$ Tween 20 (PBST) at room temperature for $30 \mathrm{~min}$, and incubated in $70 \%$ ethanol overnight at $4{ }^{\circ} \mathrm{C}$. Staining was performed with $1 \mu \mathrm{M} \mathrm{Cal}$ Fluor 610 conjugated pals-5 smFISH probes (Biosearch Technologies) in smFISH hybridization buffer ( $10 \%$ formamide, $2 \times$ SSC, $10 \%$ dextran sulfate, $2 \mathrm{mM}$ vanadyl ribonucleoside complex, $0.02 \%$ RNase free BSA, $50 \mu \mathrm{g}$. coli tRNA) at $30^{\circ} \mathrm{C}$ in the dark overnight. Samples were incubated in the wash buffer $(10 \%$ formamide, $2 \times$ SSC) at $30^{\circ} \mathrm{C}$ in the dark for $30 \mathrm{~min}$. Vectashield + DAPI was added to each sample, and stained worms were transferred to microscope slides and covered with glass coverslips. Z-stacks of the body region containing anterior part of the intestine was performed using Zeiss Axio Imager M1 compound microscope with a $\times 63$ oil immersion objective. Image processing was performed using FIJI. smFISH spot quantification was performed using StarSearch program (http:// rajlab.seas.upenn.edu/StarSearch/launch.html). When selecting the region of interest, the anterior boundary of the first four intestinal cells was determined based on the prominent border between pharynx and intestine, which is visible in the DIC channel. The posterior boundary was set at the middle distance between DAPI-stained nuclei of the first and the second intestinal rings.

PALS-5 expression and anti-PALS-5 antibody synthesis. A pals-5 cDNA with $\mathrm{N}$-terminal sequence ( $5^{\prime}$-tatgcatcaccaccatcaccatgaaaatctgtattttcag- $\left.3^{\prime}\right)$ and C-terminal sequence $\left(5^{\prime}\right.$-gagagaccggccggccgatccggctgctaa- $\left.3^{\prime}\right)$ was synthesized as a FragmentGENE (GENEWIZ, Azenta Life Sciences) and cloned into BsaI-HFv2 digested into a custom vector derived from pET21a. The resulting plasmid (pBEL2159), which includes an N-terminal His-TEV-tag, was transformed into Rosetta (DE3) cells (Novagen) for protein expression. For expression, LB with carbenicillin/ chloramphenicol was inoculated with Rosetta (DE3)/pBEL2159 and grown at $37^{\circ} \mathrm{C}$ with shaking at $200 \mathrm{rpm}$. The overnight culture was diluted 1:50 in $\mathrm{LB}+$ carbenicillin/chloramphenicol and then induced by adding IPTG to a final concentration of $1 \mathrm{mM}$ at $16^{\circ} \mathrm{C}$, and allowed to shake overnight. Cells were harvested by centrifugation and resuspended in lysis buffer $(50 \mathrm{mM}$ Tris $\mathrm{pH} 8$, $300 \mathrm{mM} \mathrm{NaCl}, 10 \mathrm{mM}$ imidazole, $10 \%$ Glycerol, $1 \mathrm{mM}$ phenylmethylsulfonyl fluoride (PMSF)). Cells were lysed using the Emulsiflex-C3 cell disruptor (Avestin) and then centrifuged at $4{ }^{\circ} \mathrm{C}, 12,000 \times g$ to pellet cell debris. The pellet, containing a large amount of insoluble PALS-5, was resuspended in urea lysis buffer (100 mM $\mathrm{NaH}_{2} \mathrm{PO}_{4} / 10 \mathrm{mM}$ Tris base, $10 \mathrm{mM}$ imidazole, $8 \mathrm{M}$ urea [titrated to $\mathrm{pH} 8$ with $\mathrm{NaOH}]$ ). The solubilized pellet was centrifuged at $4000 \times g$, and the supernatant collected. PALS-5 from the resulting supernatant was passed twice through NiNTA resin (Cytiva \#17531802), which was subsequently washed with urea wash buffer (100 mM NaH2PO4/10 mM Tris base, $40 \mathrm{mM}$ imidazole, $8 \mathrm{M}$ urea [titrated to $\mathrm{pH} 8$ with $\mathrm{NaOH}]$ ), and the bound proteins were then eluted in urea elution buffer ( $100 \mathrm{mM} \mathrm{NaH} \mathrm{PO}_{4} / 10 \mathrm{mM}$ Tris base, $300 \mathrm{mM}$ imidazole, $8 \mathrm{M}$ urea [titrated to pH 8 with $\mathrm{NaOH}])$. Fractions containing PALS- 5 were pooled, concentrated and dialyzed into dialysis buffer (PBS $\left(137 \mathrm{mM} \mathrm{NaCl}, 2.7 \mathrm{mM} \mathrm{KCl}, 1.5 \mathrm{mM} \mathrm{KH}_{2} \mathrm{PO}_{4}\right.$, $8.1 \mathrm{mM} \mathrm{Na} \mathrm{HPO}_{4}$ ), 3.9 M urea [titrated to $\mathrm{pH} 8$ by $\mathrm{NaOH}$ ]) overnight at room temperature. The following day, the sample was dialyzed once again in fresh dialysis buffer for $3 \mathrm{~h}$ at room temperature. The dialyzed sample was supplemented with $10 \%$ glycerol, flash frozen in liquid nitrogen for storage, and submitted to ProSci Inc. for custom antibody production (Poway, CA). Rabbits were initially immunized with $200 \mu \mathrm{g}$ full-length His::TEV tagged PALS-5 antigen in Freund's Complete Adjuvant. Rabbits were then subsequently boosted with four separate immunizations of $100 \mu \mathrm{g}$ antigen in Freund's Incomplete Adjuvant over a 16-week period. Approximately $25 \mathrm{ml}$ of serum was collected and PALS-5 polyclonal antibody was purified with an immuno-affinity chromatography column by crosslinking PALS-5 to cyanogen bromide $(\mathrm{CNBr})$-activated Sepharose $4 \mathrm{~B}$ gel. Antibody was eluted from the affinity column in $100 \mathrm{mM}$ glycine buffer $\mathrm{pH} 2.5$, precipitated with polyethylene glycol (PEG), and concentrated in PBS pH $7.4+0.02 \%$ sodium azide. Antibody concentration was determined by ELISA and used in western blot analysis described below.

Western blot analysis. $3000 \mathrm{~L} 4$ animals were treated with bortezomib or DMSO for $4 \mathrm{~h}$ at $20^{\circ} \mathrm{C}$, and then collected and washed with M9. $20 \mu \mathrm{l}$ of $6 \times$ loading buffer (375 mM Tris. $\mathrm{HCl}$ pH 6.8, $600 \mathrm{mM}$ DTT, $12 \%$ SDS, $0.06 \%$ bromophenol blue, and $60 \%$ glycerol) were added to the final sample volume of $100 \mu \mathrm{l}$. Samples were boiled at $100{ }^{\circ} \mathrm{C}$ for $10 \mathrm{~min}$ and stored at $-30^{\circ} \mathrm{C}$. Proteins were separated on a $10 \%$ sodium dodecyl sulfate-polyacrylamide gel electrophoresis precast gel (Bio-Rad), and transferred onto polyvinylidene difluoride (PVDF) membrane. Five percent nonfat dry milk in PBST was used to block for nonspecific binding for $2 \mathrm{~h}$ at room temperature. The membranes were incubated with primary antibodies overnight at $4{ }^{\circ} \mathrm{C}$ (rabbit anti-PALS-5 diluted 1:1,000 and mouse anti-tubulin (Sigma, catalog number T9026) diluted 1:3000 in blocking buffer). Next, the membranes were washed five times in PBST, and then incubated in horseradish peroxidaseconjugated secondary antibodies at room temperature for $2 \mathrm{~h}$ (goat anti-rabbit (MilliporeSigma, catalog number 401315) and goat anti-mouse (MilliporeSigma, catalog number 401215) diluted 1:10,000 in blocking buffer). After five washes in PBST, the membranes were treated with enhanced chemiluminescence (ECL) reagent (Amersham) for $5 \mathrm{~min}$, and imaged using a Chemidoc XRS + with Image Lab software (Bio-Rad). Quantification of band intensities in 3 western blot replicates was performed using Image Lab software (Bio-Rad). PALS-5 band intensities for each sample were normalized to the ratio of the tubulin expression levels between N2 DMSO control and a given sample.

RNA isolation. Total RNA isolation was performed as previously described ${ }^{15}$. Animals were washed off plates using M9, then washed with M9 and collected in TRI reagent (Molecular Research Center, Inc.). RNA was isolated using BCP phase separation reagent, followed by isopropanol and ethanol washes. For RNA-seq analysis, samples were additionally purified using RNeasy Mini kit from Qiagen.

qRT-PCR analyses. qRT-PCR analysis was performed as previously described ${ }^{15}$. In brief, cDNA was synthesized from total RNA using iScript cDNA synthesis kit (Bio-Rad). qPCR was performed using iQ SYBR Green Supermix (Bio-Rad) with the CFX Connect Real-Time PCR Detection System (Bio-Rad). At least three independent experimental replicates were performed for each qRT-PCR analysis. Each sample was analyzed in technical duplicates. All values were normalized to expression of $s n b-1$ control gene, which does not change expression upon IPR activation. The Pffafl method was used for data quantification ${ }^{68}$. The sequences of the primers used in all qRT-PCR experiments are given in the Supplementary Table 3 (primers 12-33).

RNA-seq analysis. cDNA library preparation and single-end sequencing was performed at the Institute for Genomic Medicine at the University of California, San Diego. Reads were mapped to C. elegans WS235 genome using Rsubread in RStudio (Supplementary Table 4, Supplementary Data 4). Differential expression analyses were performed using limma-voom function in Galaxy platform (https:// usegalaxy.org/). Genes with counts number lower than 0.5 counts per million (CPM) for $30 \mathrm{~min}$ timepoint samples and $1 \mathrm{CPM}$ for $4 \mathrm{~h}$ timepoint samples were filtered out. Quality weights were applied in analysis of 30 min timepoint. Differentially expressed genes had adjusted $p$-value lower than 0.05 . Visualization of the mapped reads shown in the Supplementary Fig. 9 was performed using Integrative Genomics Viewer (Broad Institute) ${ }^{69}$.

\section{Analysis of enriched gene categories in zip-1-dependent gene datasets.} Annotation and visualization of genes upregulated in wild-type but not in zip1(jy13) background was performed using WormCat online tool (http:// www.wormcat.com/ $)^{26}$.

Comparisons of differentially expressed genes from different datasets. An $\mathrm{R}$ studio package GeneOverlap was used for RNA-seq datasets comparative analyses. Differentially expressed genes from RNA-seq analyses from this study were compared with relevant previously published datasets ${ }^{11,13,15,24,25,34,70-72}$. Statistical similarity between datasets was determined using Fisher's exact test. The odds ratios, Jaccard indexes and $p$-values were calculated. Total number of genes was set to 46902 . Data are represented in the contingency tables in which odds ratio and 
Jaccard index values are shown in the heat map format, whereas $p$-values are indicated numerically.

CRISPR/Cas9-mediated tagging of zip-1. A long, partially single-stranded DNA donor CRISPR-Cas9 method was employed to endogenously tag the zip-1 locus ${ }^{73}$. A single sgRNA (agcaatttgagccaagctga) was used to preassemble ribonucleoprotein with Cas9 (IDT). Repair templates that contain $g f p$, $s b p$ (Streptavidin-Binding Peptide) and 3xFlag tags were amplified from plasmid pET386 using primers 5-8 from Supplementary Table 3. Injection quality was monitored by co-injecting animals with pRF4 plasmid (rol-6(su1006) marker). PCR screening of GFP insertion was performed using primers 3,4 , and 9 from the Supplementary Table 3. A line containing $g f p:: s b p:: 3 x$ Flag insertion before endogenous $z i p-1$ stop codon was backcrossed three times to the $\mathrm{N} 2$ strain before it was used in experiments.

Tissue-specific RNAi analysis. Tissue-specific RNAi analysis was performed using the feeding method. E. coli OP50-1 strain was modified to enable $z i p-1$ RNAi or control RNAi (L4440). Bacterial overnight cultures were plated on NGM plates supplemented with $5 \mathrm{mM}$ IPTG and $1 \mathrm{mM}$ carbenicillin, and incubated at room temperature for 3 or 4 days. Three thousand synchronized L1 animals were transferred to prepared plates and grown at $20^{\circ} \mathrm{C}$ for $48 \mathrm{~h}$. Animals were then treated with bortezomib or DMSO as described earlier. VP303 (rde-1) and MGH167 (sid-1) strains were used for intestinal RNAi; NR222 ( $r d e-1)$ strain was used for epidermal RNAi. Replicates that were included into analysis of sid-1 mutants had at least 50 -fold increase in pals-5 expression levels on control RNAi plates following bortezomib treatment. This threshold allowed detection of any substantial decrease in pals-5 induction in zip-1(RNAi) samples.

Killing assays. For $N$. parisii killing assays, about $150 \mathrm{~L} 1$ worms were mixed with $50 \mu \mathrm{l}$ of a $10 \times$ concentration of OP50-1 E. coli and 1 million N. parisii spores, and placed onto a $3.5 \mathrm{~cm}$ tissue culture-treated NGM plate (3 plates for each strain). After $66 \mathrm{~h}$ of infection at $25^{\circ} \mathrm{C}$, alive animals were transferred onto new NGM plates containing only OP50-1 E. coli food (30 animals per plate, 3 plates per worm strain). Animals were scored daily and alive animals were transferred to fresh NGM plates. Data from three experimental replicates were merged and analyzed using Survival function in GraphPad Prism 9; log-rank (Mantel-Cox) test was used for statistical analyses.

Longevity assays. For longevity assays, about $75 \mathrm{Ll}$ worms were mixed with $50 \mu \mathrm{l}$ of a $10 \times$ concentration of OP50-1 E. coli, and placed onto a $3.5 \mathrm{~cm}$ tissue culturetreated NGM plate ( 3 plates for each strain). After $66 \mathrm{~h}$ incubation at $25^{\circ} \mathrm{C}$, animals were transferred to new NGM plates supplemented with OP50-1 E. coli food source (30 animals per plate, 3 plates per strain). Animals were scored daily and alive animals were transferred to fresh NGM plates. Data from three experimental replicates were merged and analyzed using Survival function in GraphPad Prism 9; log-rank (Mantel-Cox) test was used for statistical analyses.

Bead feeding assay. Two thousands synchronized L1 worms were mixed with $6 \mu \mathrm{l}$ fluorescent beads (Fluoresbrite Polychromatic Red Microspheres, Polysciences Inc.), $25 \mu \mathrm{l}$ 10X concentrated OP50 E. coli, 500,000 N. parisii spores and M9 (total volume $300 \mathrm{ul}$ ). This mixture was then plated on $6 \mathrm{~cm}$ NGM plates, allowed to dry for $5 \mathrm{~min}$ and then incubated at $25^{\circ} \mathrm{C}$. After $5 \mathrm{~min}$, plates were shifted to ice, washed with ice-cold PBST and fixed in $4 \%$ paraformaldehyde. Animals were imaged using ImageXpress automated imaging system Nano imager (Molecular Devices, LLC). Fluorescence was analyzed in FIJI program.

Thermotolerance assay. Animals were grown on NGM plates at $20^{\circ} \mathrm{C}$ until L4 stage. L4 animals were transferred to new plates and exposed to heat shock at $37.5^{\circ} \mathrm{C}$ for $2 \mathrm{~h}$. Recovery was performed at room temperature for $1 \mathrm{~h}$ on a single layer, followed by $24 \mathrm{~h}$ incubation at $20^{\circ} \mathrm{C}$. After this time, animals were scored for viability based on their ability to move after touch. Three plates with 30 animals per plate were analyzed for each strain. Three experimental replicates were performed.

Body length measurements. For body length analysis of wild-type and sid-1(-); vha-6p::sid-1 mutant strains (Supplementary Fig. 10b), synchronized L1 animals were placed on control or act-5 RNAi plates and allowed to grow at $20^{\circ} \mathrm{C}$ for $48 \mathrm{~h}$. For analysis of wild-type, zip-1(jy13) and pnp-1(-) mutants (Supplementary Fig. 13b), synchronized L1 animals were plated on NGM plates and allowed to grow at $20^{\circ} \mathrm{C}$ for $44 \mathrm{~h}$. Animals were washed off the plates with M9 and fixed in $4 \%$ paraformaldehyde (Supplementary Fig. 10b) or anesthetized with $10 \mu \mathrm{M}$ levamisole (Supplementary Fig. 13b). Animals were imaged using ImageXpress automated imaging system Nano imager (Molecular Devices, LLC) in 96-well plates. Length of each animal was measured using FIJI program. Fifty animals animals were analyzed for each strain, in each of three experimental replicates.
Reporting summary. Further information on experimental design is available in the Nature Research Reporting Summary linked to this paper.

\section{Data availability}

The RNA-seq reads data generated in this study have been deposited in the NCBI GEO database under accession code GSE183361. Supplementary Information file and Supplementary Data files are provided with this paper. C. elegans WS235 genome data used in RNA-seq analysis are publicly available: https://www.ncbi.nlm.nih.gov/assembly/ GCF_000002985.6) Source data are provided with this paper.

Received: 15 June 2021; Accepted: 1 December 2021; Published online: 10 January 2022

\section{References}

1. Kasuga, Y., Zhu, B., Jang, K. J. \& Yoo, J. S. Innate immune sensing of coronavirus and viral evasion strategies. Exp. Mol. Med. 53, 723-736 (2021).

2. Rehwinkel, J. \& Gack, M. U. RIG-I-like receptors: their regulation and roles in RNA sensing. Nat. Rev. Immunol. 20, 537-551 (2020).

3. Yamada, T. et al. RIG-I triggers a signaling-abortive anti-SARS-CoV-2 defense in human lung cells. Nat. Immunol. https://doi.org/10.1038/s41590-02100942-0 (2021).

4. Yin, X. et al. MDA5 governs the innate immune response to SARS-CoV-2 in lung epithelial cells. Cell Rep. 34, 108628 (2021).

5. Lowery, S. A., Sariol, A. \& Perlman, S. Innate immune and inflammatory responses to SARS-CoV-2: implications for COVID-19. Cell Host Microbe https://doi.org/10.1016/j.chom.2021.05.004 (2021).

6. Felix, M. A. et al. Natural and experimental infection of Caenorhabditis nematodes by novel viruses related to nodaviruses. PLoS Biol. 9, e1000586 (2011).

7. Ashe, A. et al. A deletion polymorphism in the Caenorhabditis elegans RIG-I homolog disables viral RNA dicing and antiviral immunity. Elife 2, e00994 (2013).

8. Gammon, D. B. et al. The antiviral RNA interference response provides resistance to lethal arbovirus infection and vertical transmission in Caenorhabditis elegans. Curr. Biol. 27, 795-806 (2017).

9. Guo, X., Zhang, R., Wang, J., Ding, S. W. \& Lu, R. Homologous RIG-I-like helicase proteins direct RNAi-mediated antiviral immunity in C. elegans by distinct mechanisms. Proc. Natl Acad. Sci. USA 110, 16085-16090 (2013).

10. Sowa, J. N. et al. The Caenorhabditis elegans RIG-I homolog DRH-1 mediates the intracellular pathogen response upon viral infection. J. Virol. https:// doi.org/10.1128/JVI.01173-19 (2020)

11. Bakowski, M. A. et al. Ubiquitin-mediated response to microsporidia and virus infection in C. elegans. PLoS Pathog. 10, e1004200 (2014).

12. Reddy, K. C. et al. An intracellular pathogen response pathway promotes proteostasis in C. elegans. Curr. Biol. 27, 3544-3553 (2017).

13. Reddy, K. C. et al. Antagonistic paralogs control a switch between growth and pathogen resistance in C. elegans. PLoS Pathog. 15, e1007528 (2019).

14. Irazoqui, J. E., Urbach, J. M. \& Ausubel, F. M. Evolution of host innate defence: insights from Caenorhabditis elegans and primitive invertebrates. Nat. Rev. Immunol. 10, 47-58 (2010).

15. Tecle, E. et al. The purine nucleoside phosphorylase pnp-1 regulates epithelial cell resistance to infection in C. elegans. PLoS Pathog. 17, el009350 (2021).

16. Leyva-Diaz, E. et al. Silencing of repetitive DNA is controlled by a member of an unusual Caenorhabditis elegans gene family. Genetics 207, 529-545 (2017).

17. Lazetic, V. \& Troemel, E. R. Conservation lost: host-pathogen battles drive diversification and expansion of gene families. FEBS J. https://doi.org/10.1111/ febs.15627 (2020)

18. Panek, J. et al. A cullin-RING ubiquitin ligase promotes thermotolerance as part of the intracellular pathogen response in Caenorhabditis elegans. Proc. Natl Acad. Sci. USA 117, 7950-7960 (2020).

19. Botts, M. R., Cohen, L. B., Probert, C. S., Wu, F. \& Troemel, E. R. Microsporidia intracellular development relies on Myc interaction network transcription factors in the host. G3 (Bethesda) 6, 2707-2716 (2016).

20. Zhang, X. et al. Antagonistic fungal enterotoxins intersect at multiple levels with host innate immune defences. PLoS Genet. 17, e1009600 (2021).

21. Dierking, K. et al. Unusual regulation of a STAT protein by an SLC6 family transporter in C. elegans epidermal innate immunity. Cell Host Microbe 9, 425-435 (2011).

22. Sfarcic, I., Bui, T., Daniels, E. C. \& Troemel, E. R. Nanoluciferase-based method for detecting gene expression in Caenorhabditis elegans. Genetics 213, 1197-1207 (2019)

23. Osman, G. A. et al. Natural infection of C. elegans by an oomycete reveals a new pathogen-specific immune response. Curr. Biol. 28, 640-648 (2018).

24. Fasseas, M. K. et al. Chemosensory neurons modulate the response to oomycete recognition in Caenorhabditis elegans. Cell Rep. 34, 108604 (2021) 
25. Oliveira, R. P. et al. Condition-adapted stress and longevity gene regulation by Caenorhabditis elegans SKN-1/Nrf. Aging Cell 8, 524-541 (2009).

26. Holdorf, A. D. et al. WormCat: An online tool for annotation and visualization of Caenorhabditis elegans genome-scale data. Genetics 214, 279-294 (2020).

27. Shih, J. D. \& Hunter, C. P. SID-1 is a dsRNA-selective dsRNA-gated channel. RNA 17, 1057-1065 (2011)

28. Watts, J. S. et al. New strains for tissue-specific RNAi studies in Caenorhabditis elegans. G3 (Bethesda) 10, 4167-4176 (2020).

29. Qadota, H. et al. Establishment of a tissue-specific RNAi system in C. elegans. Gene 400, 166-173 (2007).

30. MacQueen, A. J. et al. ACT-5 is an essential Caenorhabditis elegans actin required for intestinal microvilli formation. Mol. Biol. Cell 16, 3247-3259 (2005).

31. Segrist, E. \& Cherry, S. Using diverse model systems to define intestinal epithelial defenses to enteric viral infections. Cell Host Microbe 27, 329-344 (2020).

32. Felix, M. A. \& Wang, D. Natural viruses of Caenorhabditis nematodes. Annu. Rev. Genet. 53, 313-326 (2019).

33. Buchon, N., Silverman, N. \& Cherry, S. Immunity in Drosophila melanogaster-from microbial recognition to whole-organism physiology. Nat. Rev. Immunol. 14, 796-810 (2014).

34. Tanguy, M. et al. An alternative STAT signaling pathway acts in viral immunity in Caenorhabditis elegans. mBio https://doi.org/10.1128/ mBio.00924-17 (2017).

35. Reinke, A. W., Baek, J., Ashenberg, O. \& Keating, A. E. Networks of bZIP protein-protein interactions diversified over a billion years of evolution. Science 340, 730-734 (2013).

36. Hoeven, R., McCallum, K. C., Cruz, M. R. \& Garsin, D. A. Ce-Duox1/BLI-3 generated reactive oxygen species trigger protective SKN-1 activity via p38 MAPK signaling during infection in C. elegans. PLoS Pathog. 7, e1002453 (2011).

37. Shivers, R. P. et al. Phosphorylation of the conserved transcription factor ATF-7 by PMK-1 p38 MAPK regulates innate immunity in Caenorhabditis elegans. PLoS Genet. 6, e1000892 (2010)

38. Reddy, K. C., Dunbar, T. L., Nargund, A. M., Haynes, C. M. \& Troemel, E. R. The C. elegans CCAAT-enhancer-binding protein gamma is required for surveillance immunity. Cell Rep. 14, 1581-1589 (2016).

39. Dunbar, T. L., Yan, Z., Balla, K. M., Smelkinson, M. G. \& Troemel, E. R. C. elegans detects pathogen-induced translational inhibition to activate immune signaling. Cell Host Microbe 11, 375-386 (2012).

40. Estes, K. A., Dunbar, T. L., Powell, J. R., Ausubel, F. M. \& Troemel, E. R. bZIP transcription factor zip-2 mediates an early response to Pseudomonas aeruginosa infection in Caenorhabditis elegans. Proc. Natl Acad. Sci. USA 107, 2153-2158 (2010).

41. McEwan, D. L., Kirienko, N. V. \& Ausubel, F. M. Host translational inhibition by Pseudomonas aeruginosa Exotoxin A Triggers an immune response in Caenorhabditis elegans. Cell Host Microbe 11, 364-374 (2012).

42. Tjahjono, E. \& Kirienko, N. V. A conserved mitochondrial surveillance pathway is required for defense against Pseudomonas aeruginosa. PLoS Genet. 13, e1006876 (2017).

43. Deng, P. et al. Mitochondrial UPR repression during Pseudomonas aeruginosa infection requires the bZIP protein ZIP-3. Proc. Natl Acad. Sci. USA 116 6146-6151 (2019).

44. Pellegrino, M. W. et al. Mitochondrial UPR-regulated innate immunity provides resistance to pathogen infection. Nature 516, 414-417 (2014).

45. Dasgupta, M. et al. NHR-49 transcription factor regulates immunometabolic response and survival of Caenorhabditis elegans during Enterococcus faecalis Infection. Infect. Immun https://doi.org/10.1128/IAI.00130-20 (2020).

46. Singh, V. \& Aballay, A. Heat-shock transcription factor (HSF)-1 pathway required for Caenorhabditis elegans immunity. Proc. Natl Acad. Sci. USA 103, 13092-13097 (2006).

47. Singh, V. \& Aballay, A. Regulation of DAF-16-mediated innate immunity in Caenorhabditis elegans. J. Biol. Chem. 284, 35580-35587 (2009).

48. Visvikis, O. et al. Innate host defense requires TFEB-mediated transcription of cytoprotective and antimicrobial genes. Immunity 40, 896-909 (2014).

49. Yang, W., Dierking, K., Rosenstiel, P. C. \& Schulenburg, H. GATA transcription factor as a likely key regulator of the Caenorhabditis elegans innate immune response against gut pathogens. Zoology 119, 244-253 (2016).

50. Shapira, M. et al. A conserved role for a GATA transcription factor in regulating epithelial innate immune responses. Proc. Natl Acad. Sci. USA 103, 14086-14091 (2006).

51. Troha, K., Im, J. H., Revah, J., Lazzaro, B. P. \& Buchon, N. Comparative transcriptomics reveals CrebA as a novel regulator of infection tolerance in D. melanogaster. PLoS Pathog. 14, e1006847 (2018).

52. Myllymaki, H. \& Ramet, M. JAK/STAT pathway in Drosophila immunity. Scand. J. Immunol. 79, 377-385 (2014)

53. Xu, J. \& Cherry, S. Viruses and antiviral immunity in Drosophila. Dev. Comp. Immunol. 42, 67-84 (2014)
54. Hedengren-Olcott, M. et al. Differential activation of the NF-kappaB-like factors Relish and Dif in Drosophila melanogaster by fungi and Gram-positive bacteria. J. Biol. Chem. 279, 21121-21127 (2004).

55. Hedengren, M. et al. Relish, a central factor in the control of humoral but not cellular immunity in Drosophila. Mol. Cell 4, 827-837 (1999).

56. Hayden, M. S., West, A. P. \& Ghosh, S. NF-kappaB and the immune response Oncogene 25, 6758-6780 (2006).

57. Iwanaszko, M. \& Kimmel, M. NF-kappaB and IRF pathways: cross-regulation on target genes promoter level. BMC Genomics 16, 307 (2015).

58. Mogensen, T. H. IRF and STAT transcription factors-from basic biology to roles in infection, protective immunity, and primary immunodeficiencies. Front. Immunol. 9, 3047 (2018).

59. Zinatizadeh, M. R. et al. The Nuclear Factor Kappa B (NF-kB) signaling in cancer development and immune diseases. Genes Dis. 8, 287-297 (2021).

60. Galli, G. \& Saleh, M. Immunometabolism of -macrophages in bacterial infections. Front. Cell Infect. Microbiol. 10, 607650 (2020).

61. Gold, K. S. \& Bruckner, K. Macrophages and cellular immunity in Drosophila melanogaster. Semin. Immunol. 27, 357-368 (2015).

62. Pukkila-Worley, R. \& Ausubel, F. M. Immune defense mechanisms in the Caenorhabditis elegans intestinal epithelium. Curr. Opin. Immunol. 24, 3-9 (2012).

63. Balla, K. M., Andersen, E. C., Kruglyak, L. \& Troemel, E. R. A wild C. elegans strain has enhanced epithelial immunity to a natural microsporidian parasite. PLoS Pathog. 11, e1004583 (2015)

64. Balla, K. M., Lazetic, V. \& Troemel, E. R. Natural variation in the roles of C. elegans autophagy components during microsporidia infection. PLoS ONE 14, e0216011 (2019).

65. Emmons, S. W., Klass, M. R. \& Hirsh, D. Analysis of the constancy of DNA sequences during development and evolution of the nematode Caenorhabditis elegans. Proc. Natl Acad. Sci. USA 76, 1333-1337 (1979).

66. Arribere, J. A. et al. Efficient marker-free recovery of custom genetic modifications with CRISPR/Cas9 in Caenorhabditis elegans. Genetics 198, 837-846 (2014).

67. Paix, A., Folkmann, A., Rasoloson, D. \& Seydoux, G. High efficiency, homology-directed genome editing in Caenorhabditis elegans using CRISPRCas9 ribonucleoprotein complexes. Genetics 201, 47-54 (2015).

68. Pfaffl, M. W. A new mathematical model for relative quantification in realtime RT-PCR. Nucleic Acids Res. 29, e45 (2001).

69. Robinson, J. T. et al. Integrative genomics viewer. Nat. Biotechnol. 29, 24-26 (2011).

70. Sarkies, P., Ashe, A., Le Pen, J., McKie, M. A. \& Miska, E. A. Competition between virus-derived and endogenous small RNAs regulates gene expression in Caenorhabditis elegans. Genome Res. 23, 1258-1270 (2013).

71. Chen, K., Franz, C. J., Jiang, H., Jiang, Y. \& Wang, D. An evolutionarily conserved transcriptional response to viral infection in Caenorhabditis nematodes. BMC Genomics 18, 303 (2017).

72. Steinbaugh, M. J. et al. Lipid-mediated regulation of SKN-1/Nrf in response to germ cell absence. Elife https://doi.org/10.7554/eLife.07836 (2015).

73. Dokshin, G. A., Ghanta, K. S., Piscopo, K. M. \& Mello, C. C. Robust genome editing with short single-stranded and long, partially single-stranded DNA donors in Caenorhabditis elegans. Genetics 210, 781-787 (2018).

\section{Acknowledgements}

This work was supported by NIH under R01 AG052622 and GM114139 to E.R.T. NIGMS/NIH award K12GM068524 to S.S.G., and the American Heart Association postdoctoral award 19POST34460023 to V.L. We thank Damian Ekiert, Crystal Chhan, Eillen Tecle, and Cheng-Ju Kuo for helpful comments on the manuscript. We thank Eillen Tecle for crossing strains to create the zip-1(jy13); pnp-1; jyIs 8 strain and for performing preliminary analyses on these animals. We thank Damian Ekiert for his help with PALS-5 protein synthesis. We thank Yishi Jin and Rose Malinow for providing reagents. RNA-seq data were generated at the UC San Diego IGM Genomics Center utilizing an Illumina NovaSeq 6000 that was purchased with funding from a National Institutes of Health SIG grant (\#S10 OD026929). The models in Fig. 7 were created using BioRender.com.

\section{Author contributions}

V.L. and E.R.T. designed the study. E.R.T. supervised the study. V.L. performed the majority of experiments and data analyses. F.W. and K.C.R. performed the RNAi screens that identified $z i p-1$ as a regulator of pals-5p::GFP expression. L.B.C. created zip-1 deletion alleles $j y 13$ and jy14. Y.C. and G.B. generated the anti-PALS-5 antibody in coordination with S.S.G., who did preliminary antibody testing. V.L. made the figures, supplementary tables, and data files. V.L. and E.R.T. wrote the manuscript. All authors read the manuscript. L.B.C., G.B., and S.S.G. commented on the manuscript.

\section{Competing interests}

The authors declare no competing interests. 


\section{Additional information}

Supplementary information The online version contains supplementary material available at https://doi.org/10.1038/s41467-021-27621-w.

Correspondence and requests for materials should be addressed to Emily R. Troemel.

Peer review information Nature Communications thanks the anonymous reviewer(s) for their contribution to the peer review of this work.

Reprints and permission information is available at http://www.nature.com/reprints

Publisher's note Springer Nature remains neutral with regard to jurisdictional claims in published maps and institutional affiliations. (c) (i) Open Access This article is licensed under a Creative Commons Attribution 4.0 International License, which permits use, sharing, adaptation, distribution and reproduction in any medium or format, as long as you give appropriate credit to the original author(s) and the source, provide a link to the Creative Commons license, and indicate if changes were made. The images or other third party material in this article are included in the article's Creative Commons license, unless indicated otherwise in a credit line to the material. If material is not included in the article's Creative Commons license and your intended use is not permitted by statutory regulation or exceeds the permitted use, you will need to obtain permission directly from the copyright holder. To view a copy of this license, visit http://creativecommons.org/ licenses/by/4.0/.

(C) The Author(s) 2022 